\title{
Multiwavelength study of 20 jets that emanate from the periphery of active regions ${ }^{\star}$
}

\author{
Sargam M. Mulay ${ }^{1}$, Durgesh Tripathi ${ }^{2}$, Giulio Del Zanna ${ }^{1}$, and Helen Mason ${ }^{1}$ \\ 1 DAMTP, Centre for Mathematical Sciences, University of Cambridge, Wilberforce Road, Cambridge, CB3 OWA, UK \\ e-mail: smm96@cam.ac.uk \\ 2 Inter-University Centre for Astronomy and Astrophysics, Ganeshkhind, 411007 Pune, India
}

Received 29 September 2015 / Accepted 27 January 2016

\section{ABSTRACT}

\begin{abstract}
Aims. We present a multiwavelength analysis of $20 \mathrm{EUV}$ jets which occurred at the periphery of active regions close to sunspots. We discuss the physical parameters of the jets and their relation with other phenomena such as $\mathrm{H} \alpha$ surges, nonthermal type-III radio bursts and hard X-ray (HXR) emission.

Methods. These jets were observed between August 2010 and June 2013 by the Atmospheric Imaging Assembly (AIA) instrument that is onboard the Solar Dynamic Observatory (SDO). We selected events that were observed on the solar disk within $+/-60^{\circ}$ latitude. Using AIA wavelength channels that are sensitive to coronal temperatures, we studied the temperature distribution in the jets using the line of sight (LOS) differential emission measure (DEM) technique. We also investigated the role of the photospheric magnetic field using the LOS magnetogram data from the Helioseismic and Magnetic Imager (HMI) onboard SDO.

Results. It has been observed that most of the jets originated from the western periphery of active regions. Their lifetimes range from 5 to 39 min with an average of $18 \mathrm{~min}$ and their velocities range from 87 to $532 \mathrm{~km} \mathrm{~s}^{-1}$ with an average of $271 \mathrm{~km} \mathrm{~s} \mathrm{~s}^{-1}$. All the jets are co-temporally associated with $\mathrm{H} \alpha$ surges. Most of the jets are co-temporal with nonthermal type-III radio bursts observed by the Wind/WAVES spacecraft in the frequency range from $20 \mathrm{kHz}$ to $13 \mathrm{MHz}$. We confirm the source region of these bursts using the potential field source surface (PFSS) technique. Using Reuven Ramaty High Energy Solar Spectroscopic Imager (RHESSI) observations, we found that half of the jets produced HXR emission and they often shared the same source region as the HXR emission $(6-12 \mathrm{keV})$. Ten out of 20 events showed that the jets originated in a region of flux cancellation and six jets in a region of flux emergence. Four events showed flux emergence and then cancellation during the jet evolution. DEM analyses showed that for most of the spires of the jets, the DEM peaked at around $\log T[\mathrm{~K}]=6.2 / 6.3(\sim 2 \mathrm{MK})$. In addition, we derived an emission measure and a lower limit of electron density at the location of the spire (jet $1: \log \mathrm{EM}=28.6, N_{\mathrm{e}}=1.3 \times 10^{10} \mathrm{~cm}^{-3}$; jet $2: \log \mathrm{EM}=28.0$, $N_{\mathrm{e}}=8.6 \times 10^{9} \mathrm{~cm}^{-3}$ ) and the footpoint (jet $1-\log \mathrm{EM}=28.6, N_{\mathrm{e}}=1.1 \times 10^{10} \mathrm{~cm}^{-3}$; jet $2: \log \mathrm{EM}=28.1, N_{\mathrm{e}}=8.4 \times 10^{9} \mathrm{~cm}^{-3}$ ). These results are in agreement with those obtained earlier by studying individual active region jets.

Conclusions. The observation of flux cancellation, the association with HXR emission and emission of nonthermal type-III radio bursts, suggest that the initiation and therefore, heating is taking place at the base of the jet. This is also supported by the high temperature plasma revealed by the DEM analysis in the jet footpoint (peak in the DEM at $\log T[\mathrm{~K}]=6.5$ ). Our results provide substantial constraints for theoretical modeling of the jets and their thermodynamic nature.
\end{abstract}

Key words. Sun: corona - Sun: atmosphere - Sun: transition region - Sun: UV radiation

\section{Introduction}

Solar jets are transient phenomena observed in the solar atmosphere. They appear as sharp-edged, impulsive, and collimated flows of plasma that move outwards with a bright spot at the footpoint, which forms an "inverted-Y" topology of magnetic field lines. They are observed throughout the atmosphere i.e. in the photosphere (H $\alpha$, Ca II K surges), chromosphere (UV), transition region (EUV) and corona (X-ray). Jets can occur in different environments such as coronal holes (CHs; Young \& Muglach 2014a,b), and active regions (ARs; Innes et al. 2011; Chen et al. 2013; Schmieder et al. 2013; Chandra et al. 2015), where they have different manifestations.

Numerous studies have been dedicated to the behaviour and different properties of jets such as length, width, lifetime, velocity etc (see e.g., Shimojo et al. 1996; Shimojo \& Shibata 2000; Schmieder et al. 2013). The statistical study of X-ray jets (Shimojo et al. 1996) observed by Soft X-ray Telescope (SXT; Tsuneta et al. 1991) onboard Yohkoh (Ogawara et al. 1991)

* Movies are available in electronic form at http: //www . aanda.org showed that jets most often occur in the western periphery of the leading spot. Other high-resolution observations showed that jets that occur at the periphery of the active regions/sunspots are mostly associated with a nonthermal type-III radio burst which is known to be produced by energetic electrons that gyrate along the open magnetic field lines (Kundu et al. 1995; Raulin et al. 1996; Innes et al. 2011; Chen et al. 2013; Chandra et al. 2015). Also, it has been observed that the accelerated electrons which have access to open field lines produce impulsive, electron/ ${ }^{3} \mathrm{He}$-rich solar energetic particle (SEP) events in the interplanetary medium (Nitta et al. 2008; Wang et al. 2006) and the accelerated electrons which are trapped in closed field lines travel downwards, lose their energies owing to collision, and produce hard X-ray emission (HXR; Glesener et al. 2012; Chen et al. 2013).

Magnetic reconnection (Parker 1963; Petschek 1964; Yokoyama \& Shibata 1995) is the fundamental process believed to play an important role in the dynamics of solar jets (Shibata et al. 1992, 1994; Shibata 2008; Chifor et al. 2008; Kayshap et al. 2013; Chandra et al. 2015). It is widely thought that the principle formation mechanism for jets is magnetic reconnection, 
which follows magnetic flux emergence (Shibata et al. 2007; Moreno-Insertis et al. 2008). However, the detailed observations of the plasma heating and cooling, and the relationship to the photospheric magnetic field have yet to be understood. It has been observed that jets are associated with emerging field regions, magnetic cancellation (Chifor et al. 2008), and locations of photospheric-shearing motions (Shimojo et al. 1998).

A number of authors have studied temperature diagnostics of individual jets ( $\mathrm{CH}$ and $\mathrm{AR}$ ) using imaging and spectroscopic observations. The temperatures of jets have been studied by using two techniques that are based on different approaches - the filter-ratio method which assumes a single temperature along the line of sight (LOS; e.g., Shimojo \& Shibata 2000; Nisticò et al. 2011; Madjarska 2011; Madjarska et al. 2012; Matsui et al. 2012; Pucci et al. 2013; Young \& Muglach 2014a) and the differential emission measure (DEM) method which assumes a multi-thermal plasma along the LOS (e.g., Doschek et al. 2010; Chandrashekhar et al. 2014; Kayshap et al. 2013; Chen et al. 2013; Sun et al. 2014).

Nisticò et al. (2011) report the temperature of $\mathrm{CH}$ jets as ranging from 0.8 to $1.3 \mathrm{MK}$ using the filter-ratio method applied to STEREO/SECCHI data; whereas Pucci et al. (2013) find the temperature of the jet to be $1.7 \mathrm{MK}$. Using a DEM analysis, Doschek et al. (2010) find the temperature of a $\mathrm{CH}$ jet was $\log T[\mathrm{~K}]=6.15$; whereas Chandrashekhar et al. (2014) report a temperature of about $\log T[\mathrm{~K}]=5.89$. The presence of Fe XIV and $\mathrm{Fe} \mathrm{XV}$ emission during the jet event in EIS/Hinode observations confirm the temperature of $\mathrm{CH}$ jets of 2-3 $\mathrm{MK}$ in an analysis by Culhane et al. (2007). Recently, Madjarska (2011) showed the presence of high temperatures up to $12 \mathrm{MK}$ in the footpoints of the jet, together with a density of $4 \times 10^{10} \mathrm{~cm}^{-3}$.

Using AIA/SDO observations, Kayshap et al. (2013) studied the emission measure and temperature distribution of a surge, which was observed on the edge of an active region. Using the automated method developed by Aschwanden et al. (2013), the authors find the average temperature and a density of $2 \mathrm{MK}$ and $4.1 \times 10^{9} \mathrm{~cm}^{-3}$, respectively during the maximum rise of the surge. Also, Chen et al. (2013) studied the temperature structure of an AR jet using the same DEM method and find hot plasma $\sim 7 \mathrm{MK}$ at the footpoint of jet.

Zhang \& Ji (2014) investigate the temperature of plasma blobs that were observed along the spire of a recurrent AR jet using the DEM method, "xrt_dem_iterative2.pro", along with Monte Carlo (MC) simulations (details in Cheng et al. 2012). The authors find the DEM-weighted average temperatures of the three blobs to range from 0.5 to $4 \mathrm{MK}$ with a median value of $\sim 2.3 \mathrm{MK}$, and the densities of $3.3,1.9$, and $2.1 \times 10^{9} \mathrm{~cm}^{-3}$, respectively. Using simultaneous imaging observation from XRT and spectroscopic observations from EIS, Chifor et al. (2008) observed an AR jet component over a range of temperatures between $\log T[\mathrm{~K}]=5.4$ and 6.4 and the base of the jet was observed to be around $\log T[\mathrm{~K}]=6.2$. The authors also measured the electron density in the high velocity up-flow component using the Fe XII line ratios and find them to be above $\log N_{\mathrm{e}}\left(\mathrm{cm}^{-3}\right)=11$. Matsui et al. (2012) studied the relationship between the velocity and temperature of an AR jet using EIS and STEREO observations. The authors observed the jet structure over a wide temperature range from $\log T[\mathrm{~K}]=4.9$ to 6.4 and investigated the doppler velocities.

The cooler counterparts of hot jets (X-ray) are often observed as dark, absorbing features (Roy 1973) in the $\mathrm{H} \alpha$ observations at chromospheric temperatures called "H $\alpha$ surges" (Chae et al. 1999; Jiang et al. 2007; Schmieder et al. 1995; Canfield et al. 1996). Also, Yokoyama \& Shibata $(1995,1996)$ find both hot and cool jets along the oblique field line in 2D simulations of flux emergence. Almost all the previous studies seem to support an association between X-ray jets, EUV jets, and $\mathrm{H} \alpha$ surges in regions of the evolving magnetic field, which observed at photospheric heights. These observations seem to indicate that newly emerging flux, which appears from below, interacts with pre-existing magnetic flux in the different layers of the atmosphere and produces jet-like structures. However, details of the processes have not yet been determined. This is largely due to inadequate spatial and temporal resolution in the observations. To study the local plasma parameters (temperature, density, abundance, flows, magnetic field etc.) involved in the process of jet phenomena, the study would require simultaneous multiwavelength imaging and spectroscopic observations with very high spatial resolution and high cadence.

In this paper, we report multiwavelength observations of 20 EUV jets that were observed at the periphery of ARs during the search period August 2010 to June 2013 (see Table 1). We take advantage of the high-cadence (12s) SDO/AIA observations to characterise the jets. These kinds of high cadence observations are necessary to study these dynamic events. We have carried out the first comprehensive study of multiwavelength observations of active region jets. We used the regularized inversion technique developed by Hannah \& Kontar (2012) to recover DEM. This method gave us a better representation than the above-mentioned methods to investigate the temperature structure in the AR jets. We compute reliable estimates of errors for the DEM over the range of temperatures, which also allowed us to investigate the temperature variation of the coronal plasma for different regions in the jet events. We performed a detailed analysis of the temperature distribution for the so-called "spire" and "footpoint" regions of two jets, which has not been done in previous studies of individual AR jets. In addition, we derived a lower limit of the electron density from these results. We found significant and important differences in the physical parameters for these different regions of the jets.

In Sect. 2, we present information about the instruments that we used for this study and the analysis techniques. In Sect. 3, we discuss the characteristic behaviour of active region jets and their association with other phenomena that occurs in the solar atmosphere such as nonthermal type-III radio bursts, HXR emission, soft X-ray flares and $\mathrm{H} \alpha$ surges. In Sect. 4, we discuss the DEM analysis of two active region jets and investigate their temperature structure and electron densities at the location of the spire and at the footpoint. The discussion and summary is presented in Sect. 5.

\section{Observations}

We selected 20 EUV active region jets observed between August 2010 and June 2013 by the Atmospheric Imaging Assembly (AIA; Lemen et al. 2012) instrument onboard the Solar Dynamic Observatory (SDO). We chose these events using the daily SDO movies ${ }^{1}$ and the automatic solar feature detection tool - Heliophysics Event Knowledgebase (HEK; Hurlburt et al. $2012)^{2}$. HEK is a system which catalogues the interesting solar events and features using feature-detection methods and presents a short description of them.

In this study, we included events that satisfy the following conditions:

- the jets were associated with an active region;

\footnotetext{
1 http://sdo.gsfc.nasa.gov/data/dailymov.php

2 http://www.lmsal.com/isolsearch
} 
Table 1. Statistically measured physical parameters of 20 EUV active region jets.

\begin{tabular}{|c|c|c|c|c|c|c|c|c|c|c|c|}
\hline Date & $\begin{array}{c}\text { Time (UT) } \\
\text { in } 193 \AA\end{array}$ & $\begin{array}{r}\text { Lifetime } \\
\text { (in min) }\end{array}$ & $\begin{array}{l}\text { Active } \\
\text { region }\end{array}$ & $\begin{array}{c}\text { Sunspot } \\
\text { configuration }\end{array}$ & $\begin{array}{l}\text { GOES X-ray flare } \\
\text { Start time (UT) }\end{array}$ & $\begin{array}{l}\text { Type III } \\
\text { (UT) }\end{array}$ & $\begin{array}{c}\text { RHESSI } \\
(\mathrm{keV})\end{array}$ & $\begin{array}{c}\text { Jet velocity } \\
\left(\mathrm{km} \mathrm{s}^{-1}\right)\end{array}$ & $\mathrm{H} \alpha$ & HMI & $\begin{array}{c}\text { DEM peak temperature } \\
\log T[\mathrm{~K}]\end{array}$ \\
\hline 2010 Aug. 02 & $17: 10-17: 35$ & 25 & 11092 (N13 E07) & $\alpha$ & B $1.8(17: 21)$ & $17: 25$ & $\mathrm{R} 1, \mathrm{R} 2$ & 236 & a & $\mathrm{FE}$ & 6.3 \\
\hline 2010 Sep. 17 & $00: 15-00: 31$ & 16 & 11106 (S20 W09) & $\beta$ & B $5.7(00: 14)$ & $00: 16$ & - & 194 & $\mathrm{~b}$ & FEC & 6.0 \\
\hline 2011 Feb. 14 & $12: 51-13: 02$ & 11 & 11158 (S20 W17) & $\beta \gamma$ & - & $12: 51$ & - & 208 & d & $\mathrm{FC}$ & 6.0 \\
\hline 2011 Feb. 16 & $13: 33-13: 42$ & 09 & 11158 (S21 W41) & $\beta \gamma \delta$ & - & $13: 33$ & $\mathrm{R} 1, \mathrm{R} 2, \mathrm{R} 3$ & 207 & d & FEC & 6.0 \\
\hline 2011 Mar. 01 & $12: 53-13: 18$ & 25 & 11165 (S21 W00) & $\beta$ & - & $12: 53$ & - & 87 & d & $\mathrm{FC}$ & 6.3 \\
\hline 2011 Mar. 07 & $21: 33-22: 12$ & 39 & 11166 (N11 E13) & $\beta \gamma$ & - & $21: 48$ & - & 203 & d & FE & 6.3 \\
\hline 2011 Oct. 17 & $19: 48-20: 04$ & 16 & 11314 (N28 W32) & $\beta \beta$ & - & $19: 49$ & - & 520 & b & $\mathrm{FC}$ & 6.3 \\
\hline 2011 Dec. 11 & $03: 17-03: 26$ & 09 & 11374 (S17 E27) & $\alpha$ & B $8.6(03: 20)$ & 03:17 & $\mathrm{R} 1, \mathrm{R} 2$ & 165 & $\mathrm{a}$ & $\mathrm{FE}$ & 6.2 \\
\hline 2011 Dec. 11 & $12: 15-12: 49$ & 34 & 11374 (S17 E27) & $\alpha$ & - & $12: 15$ & $\mathrm{R} 1, \mathrm{R} 2$ & 278 & d & FC & 6.3 \\
\hline 2011 Dec. 11 & $23: 14-23: 34$ & 20 & 11374 (S17 E27) & $\alpha$ & - & $23: 14$ & $\mathrm{R} 1, \mathrm{R} 2$ & 165 & a & $\mathrm{FC}$ & 6.3 \\
\hline 2012 Mar. 05 & $21: 51-22: 00$ & 09 & 11429 (N18 E41) & $\beta \gamma \delta$ & - & $21: 51$ & - & 532 & $\mathrm{~b}$ & FC & 6.0 \\
\hline 2012 Oct. 10 & $14: 21-14: 45$ & 24 & 11585 (S20 W43) & $\beta$ & C $2.1(14: 25)$ & $14: 31$ & R2 & 259 & d & $\mathrm{FC}$ & 6.2 \\
\hline 2013 Mar. 02 & 11:49-11:57 & 08 & 11681 (N17 W08) & $\alpha \gamma$ & B $6.0(11: 48)$ & $11: 51$ & - & 316 & d & FEC & 6.0 \\
\hline 2013 Mar. 02 & $12: 04-12: 25$ & 21 & 11681 (N17 W08) & $\alpha \gamma$ & B $6.0(11: 48)$ & 12:04 & - & 210 & d & $\mathrm{FE}$ & 6.3 \\
\hline 2013 Apr. 28 & $20: 59-21: 11$ & 12 & 11731 (N09 E23) & $\beta \gamma$ & C 1.5 (20:59) & - & - & 320 & $\mathrm{~b}$ & FEC & 6.2 \\
\hline 2013 Apr. 28 & $22: 51-22: 59$ & 08 & 11731 (N09 E23) & $\beta \gamma$ & - & - & - & 168 & b & $\mathrm{FE}$ & 6.2 \\
\hline 2013 May 04 & $23: 15-23: 49$ & 34 & 11734 (S19 W04) & $\beta \gamma \delta$ & C $1.3(23: 25)$ & $23: 15$ & $\mathrm{R} 1, \mathrm{R} 2, \mathrm{R} 3, \mathrm{R} 4$ & 283 & $\mathrm{~b}$ & FC & 6.3 \\
\hline 2013 May 25 & 08:42-08:55 & 13 & 11748 (N12 W83) & $\beta \gamma \delta$ & - & - & $\mathrm{R} 1, \mathrm{R} 2$ & 322 & d & $\mathrm{FE}$ & 6.2 \\
\hline 2013 Jun. 17 & 08:41-09:06 & 25 & $11770($ S13 E13) & $\alpha$ & - & 08:52 & $\mathrm{R} 1, \mathrm{R} 2, \mathrm{R} 3$ & 409 & $\mathrm{c}$ & $\mathrm{FC}$ & 6.2 \\
\hline 2013 Jun. 18 & $15: 13-15: 39$ & 26 & 11770 (S14 E02) & $\alpha$ & - & $15: 13$ & $\mathrm{R} 1, \mathrm{R} 2, \mathrm{R} 3, \mathrm{R} 4$ & 338 & b & $\mathrm{FC}$ & 6.2 \\
\hline
\end{tabular}

Notes. $\mathrm{H} \alpha$ Surge visible in a: Solar Magnetic Activity Research Telescope (SMART) Hida Observatory; b: Big Bear Solar Observatory (BBSO); c: Kanzelhöhe Observatory; d: Global Oscillation Network Group (GONG) data; R1: RHESSI 3-6 keV; R2: RHESSI 6-12 keV; R3: RHESSI 12-25 keV; R4: RHESSI 25-50 keV; FE: Flux Emergence; FEC: Flux Emergence and then Cancellation; FC: Flux Cancellation.

- the AR jets were observed on the solar disk within $+/-60^{\circ}$ latitude.

In the current analysis, we used high temporal (12 s) and spatial resolution $\left(0.6^{\prime \prime}\right.$ per pixel) full disk images of the Sun from AIA/SDO in ten different UV/EUV pass bands. This covers the region from the lower chromosphere to the corona. We also used the LOS magnetogram data (with temporal resolution of $45 \mathrm{~s}$ and spatial resolution of $0.5^{\prime \prime}$ per pixel) from the Helioseismic and Magnetic Imager (HMI; Scherrer et al. 2012) onboard the SDO to investigate the possible cause of the jets.

To understand the relationship between nonthermal type-III radio bursts and jets, we used data from the WAVES (Bougeret et al. 1995) instrument onboard the Wind satellite. This has two radio receivers, RAD $1(20-1040 \mathrm{kHz})$ and RAD 2 $(1.075-13.825 \mathrm{MHz})$, which provide a dynamic spectrum in radio wavelengths and information about nonthermal bursts that have been observed in the solar atmosphere and interplanetary medium. We also examine the role of HXR that is associated with the jets using the high-resolution imaging data from the Reuven Ramaty High Energy Solar Spectroscopic Imager (RHESSI) mission (Lin et al. 2002). This provides us with detailed information on the position and structure of thermal and nonthermal HXR sources in different energy bands. To investigate the relationship between cool and hot temperature component of jets, we analysed $\mathrm{H} \alpha$ data using the ground-based observatories - Kanzelhöhe Observatory (Otruba 1999), Big Bear Solar Observatory (BBSO; Zirin 1970), the Solar Magnetic Activity Research Telescope (SMART; Ueno et al. 2004) at Hida observatory and the data from Global Oscillation Network Group (GONG). All the data were reduced and prepared using the standard routines that are available in the SolarSoft libraries and were normalized by the exposure time. We co-aligned HMI magnetograms with AIA $1700 \AA$ channel and then accordingly co-aligned other AIA channels.

We provide a detailed list of events and statistical information about measured physical parameters of the 20 EUV AR jets in Table 1. We report the observation of jets in the AIA $193 \AA$ channel and measured their lifetime (Cols. 2 and 3). We identify the active region locations along with their associated sunspot configurations (Cols. 4 and 5). We compare jet timings with GOES X-ray flares, RHESSI HXR emission and nonthermal type-III radio burst timings, listed in Cols. 6-8, respectively. The AIA $171 \AA$ filter images were used to see the fine structure of jets and to calculate the plane-of-sky velocities using the time-distance analysis technique. The results are listed in Col. 9. We investigate the relationship of the jets with $\operatorname{cool} \mathrm{H} \alpha$ surges (Col. 10) and their relationship with photospheric magnetic field topology using HMI magnetogram data (Col. 11). A DEM analysis was carried out to obtain the thermal structure of the jets and we present the details in Col. 12. We selected two jets for detailed analysis. We carried out similar analyses (as shown in Sects. 3 and 4) for other jets and summarised additional information and images in Appendix A.

\section{Data analysis and results}

In this section, we discuss the detailed analysis of two active region jets (Jet 1 and 13 in Table 1 which we call Jet 1 and 2 below) and also investigate their relationship to other phenomena.

\subsection{Jet 1: 2010 August 02}

\subsubsection{Overview and kinematics}

The AIA instrument observed a jet on 2010 August 02 that originated from the edge of AR 11092 (N13 E07). Figure 1 shows the temporal evolution of the jet in the AIA $193 \AA$ A wavelength channel. The jet appeared to evolve from the western periphery of the active region at 17:26 UT in all wavelength channels of the AIA. Figure 2 shows the multi-thermal structure of the jet spire. The jet activity started at 17:10 UT and ended at 17:35 UT. The white over-plotted box shows the field of view for the region, shown in Fig. 4. The first brightening at the footpoint was seen at 17:07 UT and as time progressed, a small loop starts to develop between the footpoint of the jet and the edge of the umbralpenumbral region of sunspot. At 17:18:43 UT, the loop started to expand and became associated with a spire of the jet. The untwisting nature of the complex, multi-threaded spire was clearly 

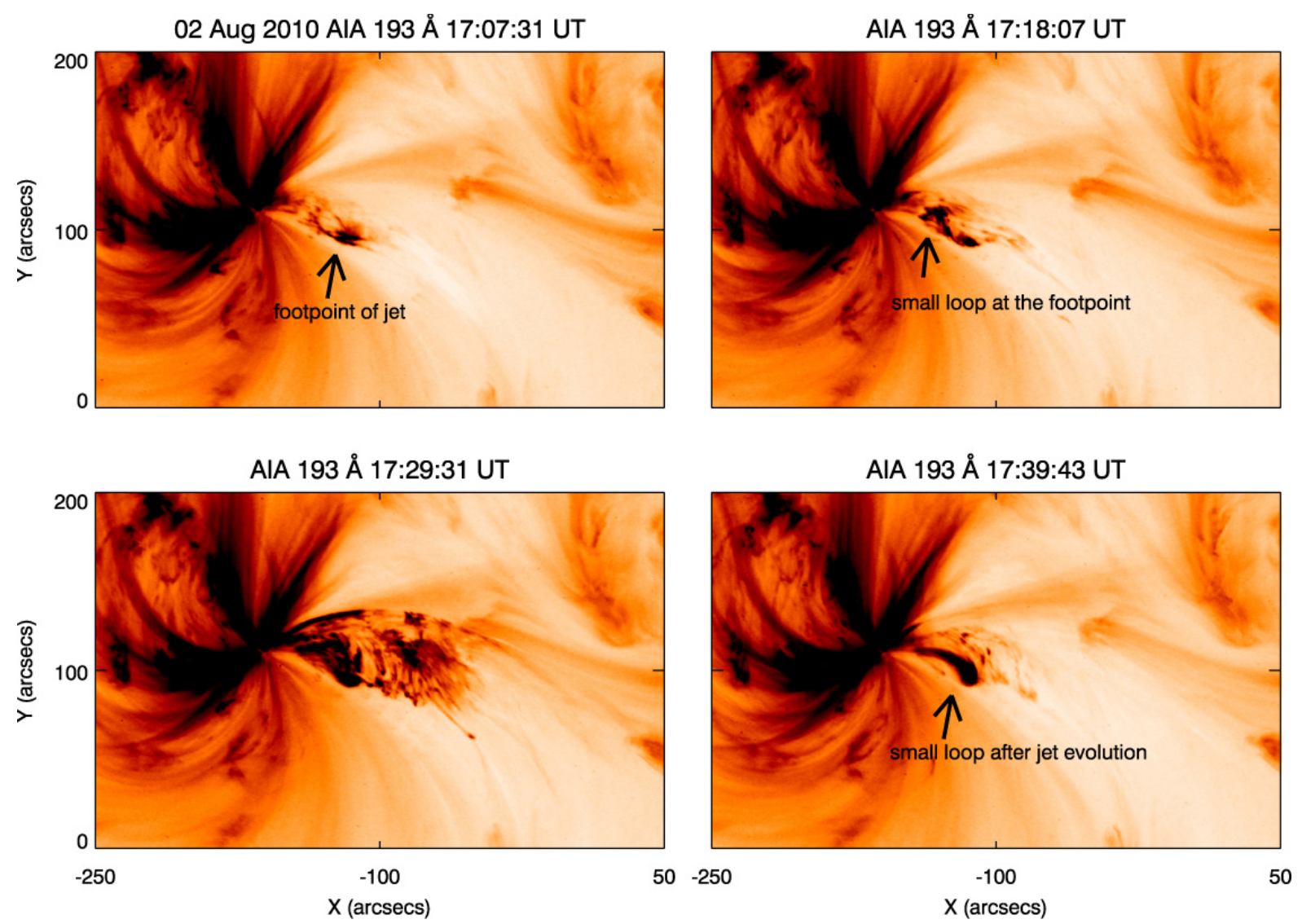

Fig. 1. Temporal evolution of the jet observed on 2010 August 2 from active region 11092 (N13 E07) in the AIA $193 \AA$ A passband. The untwisting nature of the jet plasma is seen with a small loop at the base (reverse colour image). The complex, multi-threaded spire appears to originate from the edge of the sunspot. (See online movie1.)
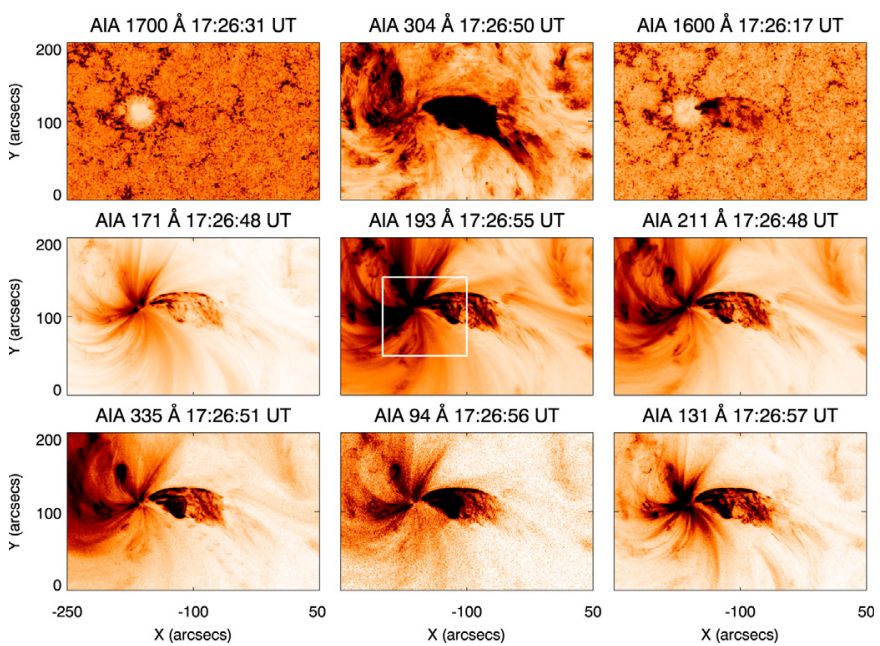

Fig. 2. Evolution of the jet observed on 2010 August 02 from active region 11092 (N13 E07) at 17:26 UT in all AIA passbands. The images show the multi-thermal structure of the jet spire originating from the edge of the sunspot (reverse colour image). The white over-plotted box shows the field of view for the region, shown in Fig. 4.

visible along with its evolution. The bright loop at the footpoint was clearly observed after the jet evolution at 17:39 UT. There was no significant $\mathrm{X}$-ray activity recorded by the Geostationary Operational Environmental Satellite (GOES) during this time.

To estimate the speed of the jet, we performed the timedistance analysis using AIA $171 \AA$ filter images (dominated by
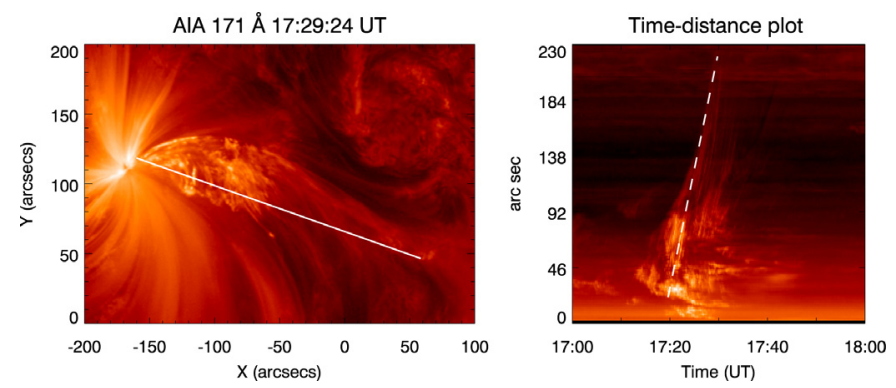

Fig. 3. Left panel: jet evolution in the AIA $171 \AA$ channel. The white line shows the artificial slit which was used to produce a time-distance plot. Right panel: time-distance plot along the jet spire. The white dashed line is used for the velocity calculation. This is found to be $236 \mathrm{~km} \mathrm{~s}^{-1}$.

Fe IX $\log T[\mathrm{~K}]=6.0)$. We employed an artificial slit along the direction of the jet spire and calculated the plane-of-sky velocity of the jet-front as shown in Fig. 3. The velocity was found to be $236 \mathrm{~km} \mathrm{~s}^{-1}$. The complex multi-threaded and untwisting nature of the jet spire is clearly visible in this plot.

\subsubsection{Photospheric magnetic field evolution}

Figure 4 shows the time evolution of the LOS component of the photospheric magnetic field in the $+/-100$ Gauss range. The active region consists of a negative-polarity sunspot and positivepolarity surrounding it. This shows an anemone structure of the magnetic field (Shibata et al. 1992, 1994). The jet was associated 

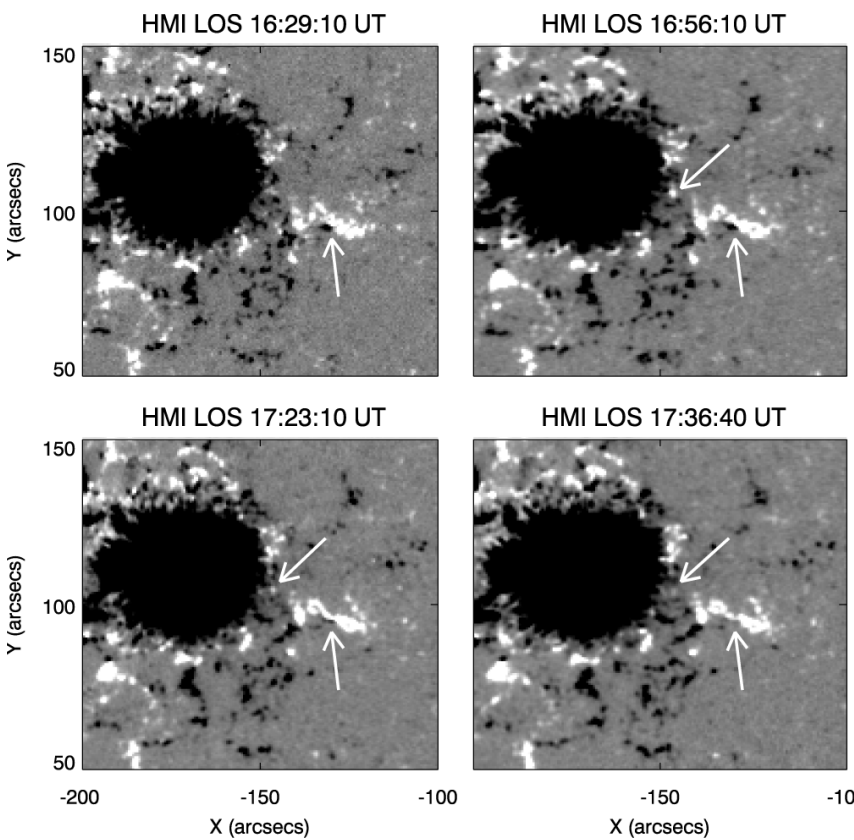

HMI LOS 17:36:40 UT

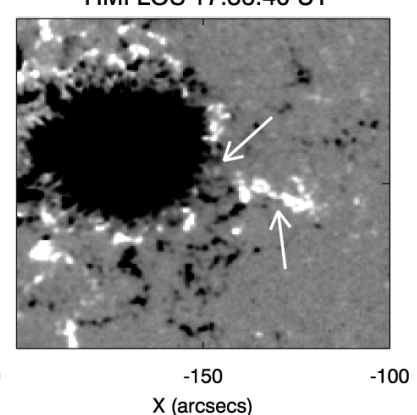

Fig. 4. HMI LOS magnetograms (+/-100 Gauss) during the jet evolution, which shows the negative-polarity sunspot (black) surrounded by positive-polarity (white). The white arrows show where there is an emergence of positive-polarity at the edge of the sunspot and negativepolarity cancellation on the western side of the sunspot during the jet evolution. (See online movie1.)

with the edge of a negative-polarity sunspot and a positivepolarity region on the western side of the sunspot as shown by white arrows in Fig. 4. This activity of magnetic field lasted for more than an hour. The evolution of positive-polarity at the edge of the sunspot indicates flux emergence while the cancellation of the negative-polarity was seen at the western side of the sunspot. This observation is co-spatial with the location of the footpoint of the jet. Figure 5 (bottom panel) shows the AIA $193 \AA$ image at 17:26 UT and the contours are from the HMI LOS at the same time. The negative-polarity sunspot is shown by blue contours, and positive-polarity as yellow contours. This image clearly shows the small loop at the footpoint of the jet evolve between the umbral-penumbral boundary of the sunspot and the nearby positive-polarity region. The region where the magnetic activity took place is shown by white arrows.

\subsection{3. $\mathrm{H} \alpha$ surge and hard X-ray emission}

We analysed the $\mathrm{H} \alpha$ data from the BBSO to investigate the presence of a low-temperature component during the jet evolution. Figure 5 (top left panel) shows the $\mathrm{H} \alpha$ image (reverse colour) of the jet at 17:26 UT. The evolution of low-temperature plasma originating from the edge of the sunspot is co-temporal and cospatial with the hot EUV jet spire plasma as seen in the AIA images. This observation indicates the multi-thermal nature of the jet spire.

To probe the spatial relationship between the jet and HXR sources, we analysed the high-resolution HXR data from the RHESSI satellite. We created images in the 3-6 and 6-12 keV energy band with the CLEAN algorithm (Hurford et al. 2002), using 4-8 sub-collimators. We integrated the HXR fluxes during the jet evolution for a few minutes to get sufficient counts. Figure 5 (top middle and right panel) shows the jet in the AIA $193 \AA$ channel at 17:23 UT and 17:26 UT and the yellow
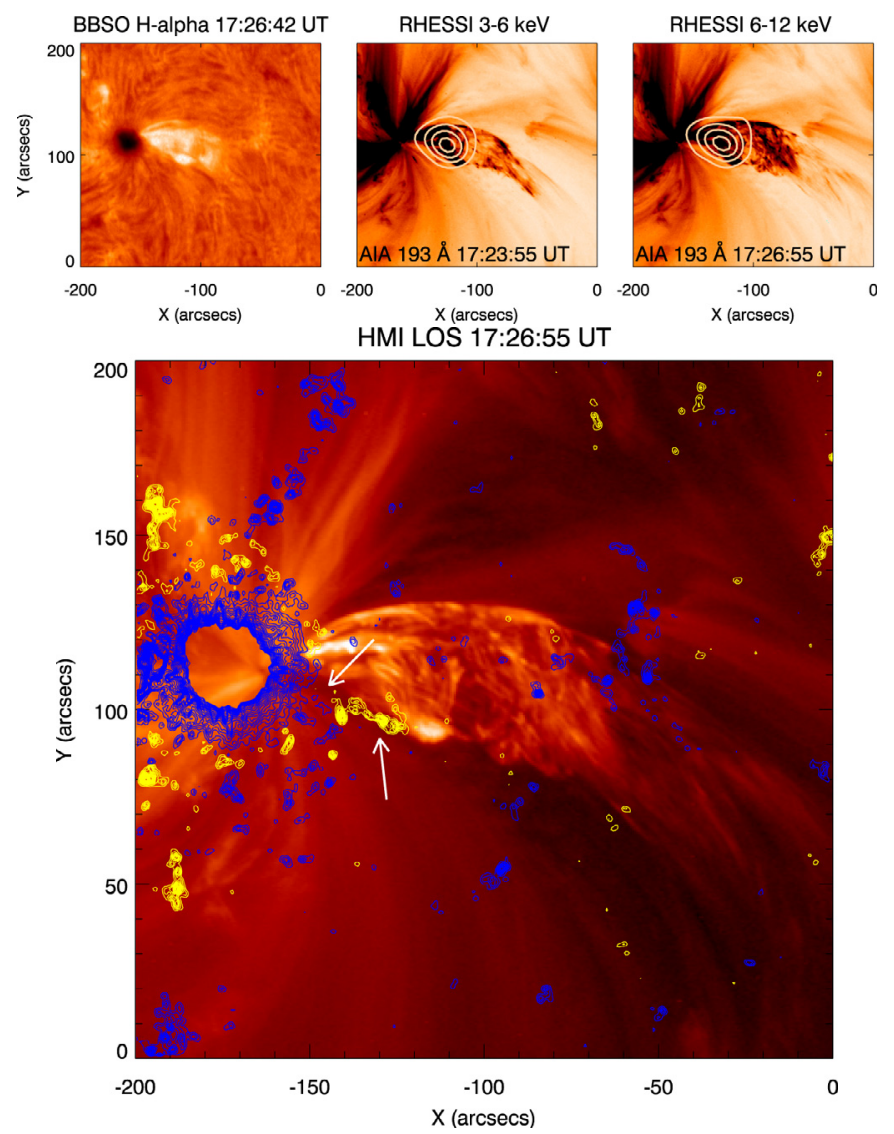

Fig. 5. Top left panel: $\mathrm{H} \alpha$ image of the jet at 17:26 UT, observed by the BBSO. Top middle and right panels: the jet in the AIA $193 \AA$ channel (reverse colour image) with 3-6 keV and 6-12 keV RHESSI contours (30, 50, 70, and 90\% of peak flux). Bottom panel: AIA 193 Å image and contours are HMI LOS at 17:26 UT. The blue colour contours represent the negative-polarity sunspot and the yellow contours show the positivepolarity regions.

contours $(30,50,70$, and $90 \%$ of the peak flux) are the HXR sources in the 3-6 and 6-12 keV energy bands respectively, integrated over 17:21-17:27 UT. The HXR sources are observed to be co-temporal and co-spatial with the footpoint of the jet.

\subsubsection{Radio emission and PFSS results}

The fast moving nonthermal electrons along the open magnetic field lines emit radio emission at the plasma frequency. This fast drifting emission appears as a pillar structure in the radio dynamic spectrum which is classified as a nonthermal type-III radio burst. These bursts provide evidence for magnetic reconnection and particle acceleration (Kundu et al. 1995; Reid \& Ratcliffe 2014; Glesener et al. 2012). We compared the jet timings with observations of dynamic spectra recorded by the WAVES instrument. A nonthermal type-III radio burst was observed at 17:25 UT by the RAD 1 and RAD 2 radio receivers in the frequency range from $13 \mathrm{MHz}$ to $220 \mathrm{kHz}$ as shown in Fig. 6 (top panel).

Furthermore, we also investigated the location of these bursts using the potential field source surface (PFSS; Schatten et al. 1969) extrapolation of the photospheric magnetic field. This tool is used to visualise the solar coronal magnetic field in the active region. Figure 6 (bottom panel) shows the extrapolated coronal magnetic field at 18:04 UT using the PFSS analysis technique. The white lines show the closed magnetic structure that is 

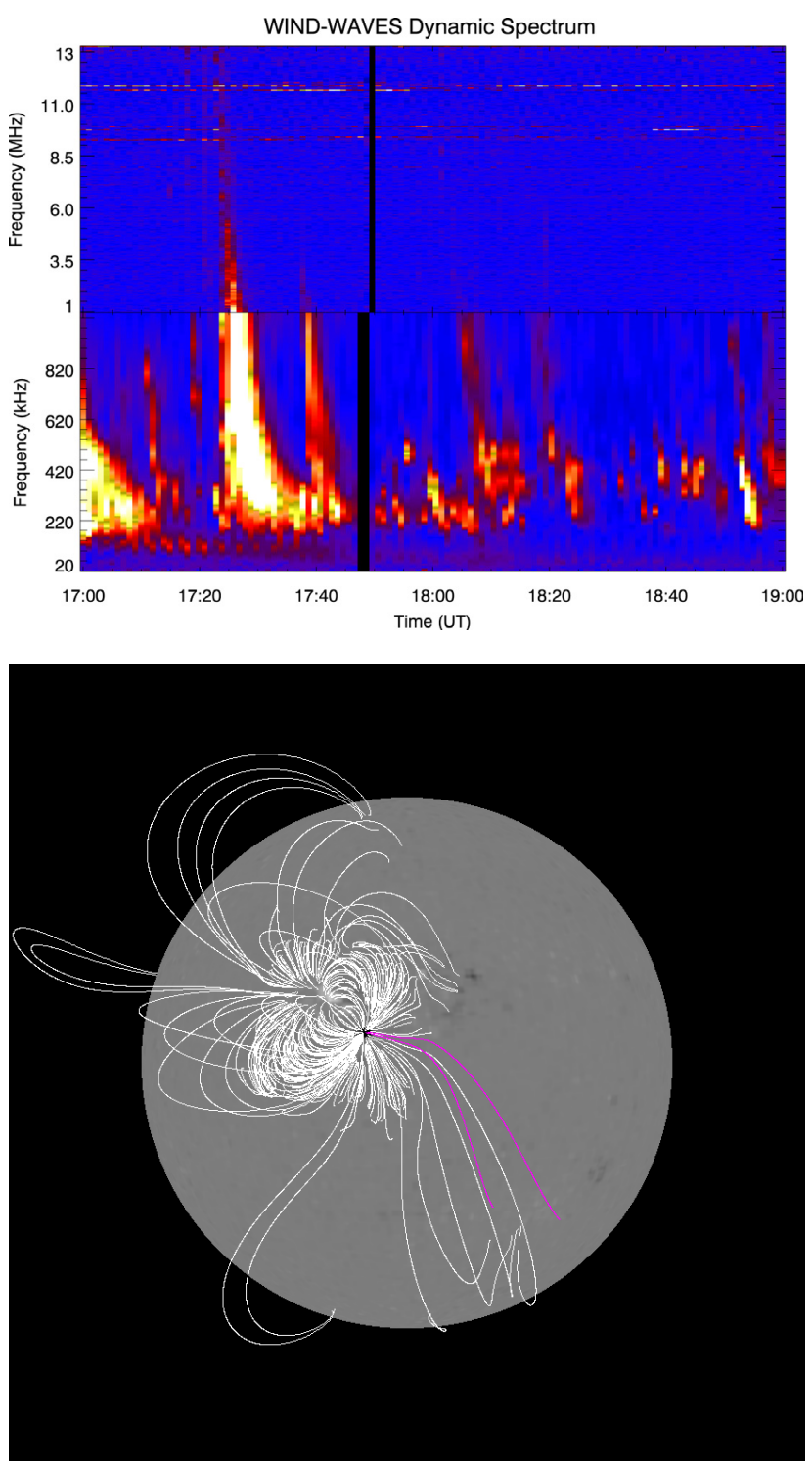

Fig. 6. Top panel: dynamic radio spectrum indicates a nonthermal type-III radio burst observed by the WIND/WAVES at 17:25 UT. This burst is co-temporal with the jet evolution. The presence of the HXR source and the nonthermal type-III radio burst indicates the signature of particle acceleration. Bottom panel: PFSS extrapolation of the active region at 18:04 UT. The white and pink lines indicate the closed and open magnetic structures in the active region and the nearby region, respectively.

associated with the active region and the pink lines indicate the open magnetic field lines. The source region of the jet and the open magnetic field lines share the same location. This indicates that the jet is ejected in the direction of these open field structures. The presence of open field lines confirm the source region of the nonthermal type-III radio burst in the jet.

\subsection{Jet 2: 2013 March 02}

\subsubsection{Overview and kinematics}

The AIA instrument observed a jet on 2013 March 02 originating from the edge of AR 11681 (N17 E41). Figure 7 shows the temporal evolution of the jet in the AIA $193 \AA$ A wavelength channel. The jet appeared to evolve from the western periphery of the active region at 11:49:43 UT in all wavelength channels of AIA, as shown in Fig. 8. The black over-plotted box shows the field of view for the region, shown in Fig. 10. A small loop-like structure was observed at the footpoint and as time progressed, it started to grow and erupt as a jet. The first brightening of the loops was seen at 11:50:18 UT, before the jet evolution. The jet activity started at 11:48 UT and ended at 12:00 UT. The continuous untwisting motion of the complex spire was clearly observed in all AIA channels. Another jet was also observed from the same location at 12:10 UT which showed a simple spire structure. This observation confirms the recurrent nature of the jet. A B6 X-ray flare was recorded at 11:48 UT by GOES.

We employed the time-distance analysis technique to calculate the plane-of-sky jet's velocity. Figure 9 (left panel) shows an image of the jet at 11:52 UT in the AIA $171 \AA$ channel and (right panel) shows the time-distance plot of the jet-front. The white line (left panel) indicates the artificial slit along the jet spire which is used to create a time-distance plot, and the white dashed line (right panel) which is used for the velocity calculation, and which is found to be $316 \mathrm{~km} \mathrm{~s}^{-1}$. The complex, multithreaded nature of the jet spire and its untwisting nature was well observed.

\subsubsection{Photospheric magnetic field evolution}

Figure 10 shows the time evolution of the LOS component of the photospheric magnetic field in the $+/-100$ Gauss range. The active region consists of a negative-polarity region. We clearly observed the positive-flux emergence and cancellation in this region which is shown by the white arrows in Fig. 10. These regions are co-spatial with the footpoint of the jet. This activity of the magnetic field lasts for half an hour. This observation indicates that the positive flux emergence and cancellation at the footpoint might have contributed to the plasma ejection.

\subsubsection{Radio emission and PFSS results}

The WAVES instrument recorded a nonthermal type-III radio burst during the jet evolution. Figure 11 (top panel) shows the radio dynamic spectrum of a nonthermal type-III radio burst at 11:50 UT recorded by the RAD $1(20-1040 \mathrm{kHz})$ and RAD 2 (1.075-13.825 MHz) receivers on the WAVES instrument.

Figure 11 (bottom panel) shows the extrapolated coronal magnetic field at 12:04 UT using the PFSS analysis technique. The white lines show the closed magnetic structure associated with the active region and the pink and green lines indicate the open magnetic field lines. The source region of the jet and the open magnetic field lines share the same location, which indicates that the jet was ejected in the direction of these open field structures. The presence of open field lines confirm the source region of nonthermal type-III radio burst.

\section{Differential emission measure (DEM)}

In this section, we discuss the DEM analysis of the two active region jets, described in Sect. 3 and investigate their temperature and electron-density structure at the location of the spire and at the footpoint.

Using the six AIA wavelength channels (94, 131, 171, 193, 211, and $335 \AA$ ), which are sensitive to a range of coronal temperatures, we performed the LOS DEM analysis by using the method of regularized inversion that was developed by Hannah \& Kontar (2012). See O’Dwyer et al. (2010), Del Zanna et al. (2011), Del Zanna (2013) for a detailed description of the EUV filters in AIA and their temperature responses. 
S. M. Mulay et al.: Multiwavelength study of active region jets
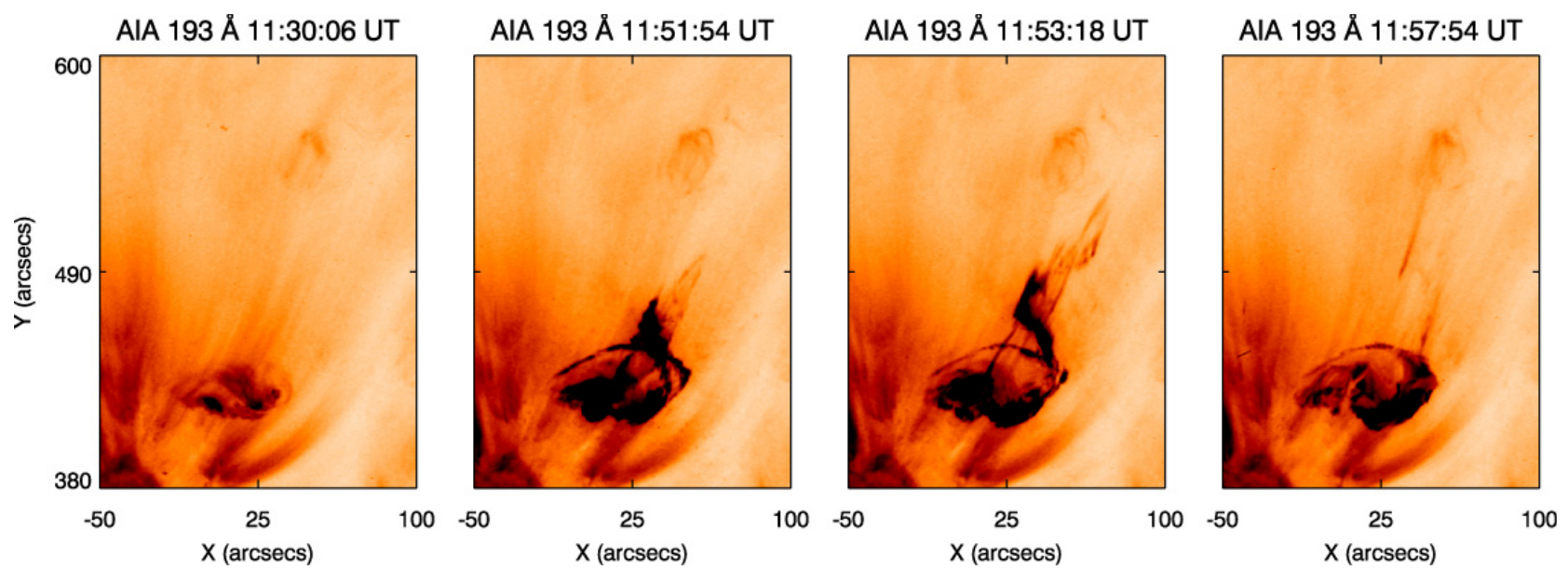

Fig. 7. Temporal evolution of a jet observed on 2013 March 02 from active region AR 11681 (N17 E41) in the AIA $193 \AA$ A passband. The complex, multi-threaded spire and a small loop at the footpoint before the jet evolution was clearly observed (reverse colour image). The complex spire shows continuous untwisting motion. (See online movie2.)
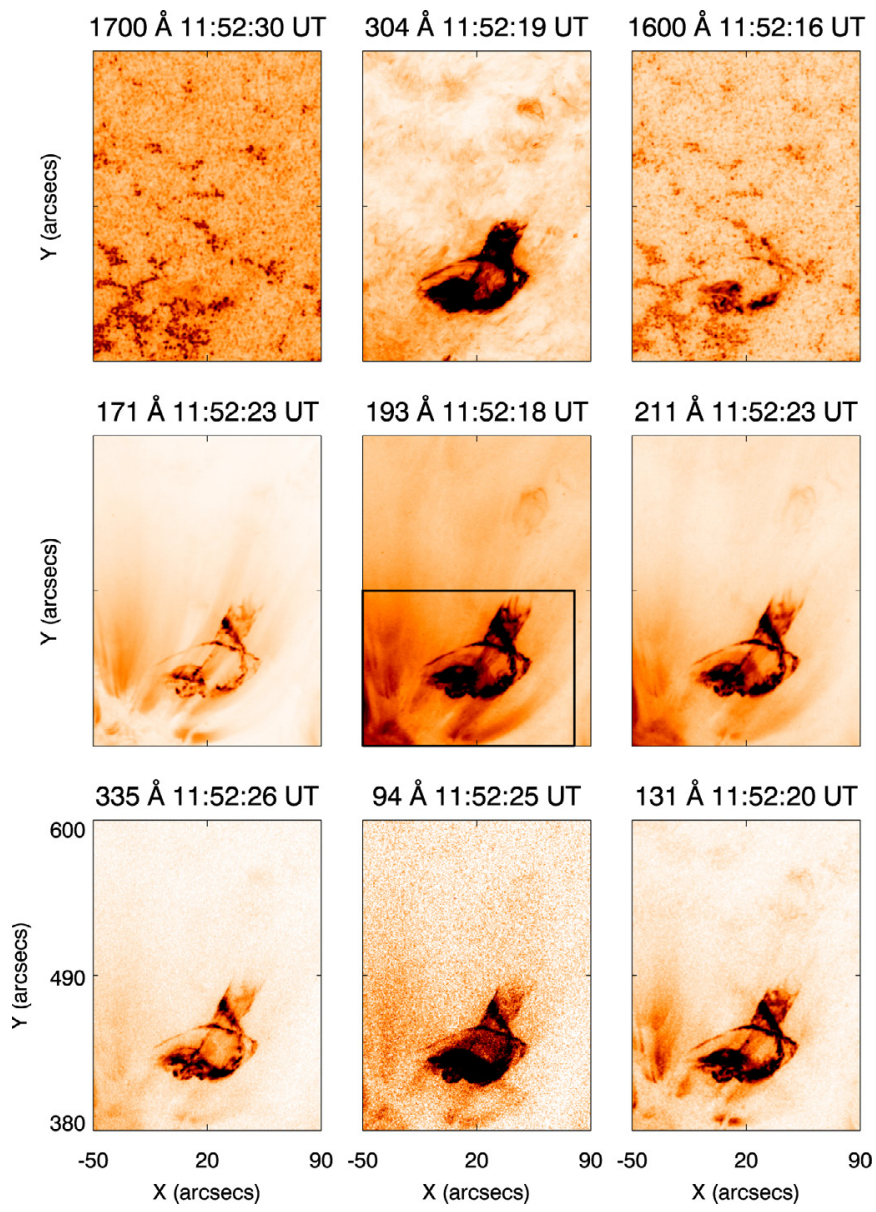

Fig. 8. Evolution of the jet on 2013 March 02 at 11:52 UT in all AIA passbands. The images show the multi-thermal structure of the jet spire originating from the edge of the sunspot (reverse colour image). The black over-plotted box shows the field of view for the region shown, in Fig. 10.

The unsaturated AIA images during the pre-jet and jet phase were used for the reconstruction of DEM maps for selected areas. The method returns a regularized DEM as a function of T. For the inversion, we used the zeroth-order regularization in a range of temperature $\log T[\mathrm{~K}]=5.5$ to $\log T[\mathrm{~K}]=7.0$
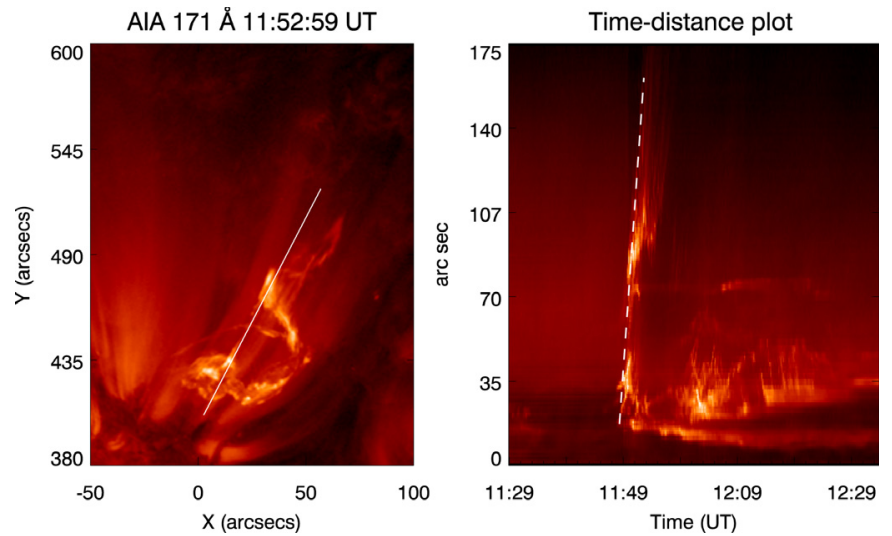

Fig. 9. Left panel: jet evolution in the AIA $171 \AA$ A channel, The white line shows an artificial slit which is used to produce the time-distance plot. Right panel: time-distance plot along the jet spire. The white dashed line was used for the plane-of-sky velocity calculation, which is found to be $316 \mathrm{~km} \mathrm{~s}^{-1}$.
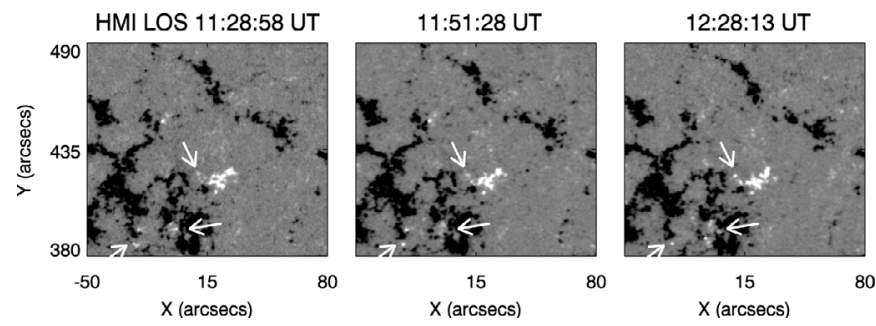

Fig. 10. HMI LOS magnetograms during the jet evolution show the positive flux emergence (white) in the negative-polarity (black) and then cancellation. The white arrows show that there is an emergence of positive-polarity during the jet evolution. (See online movie2.)

with 15 temperature bins, i.e. $\triangle \log T=0.1$ intervals. The latest version of the AIA filter response function, including the new CHIANTI v.8 (Del Zanna et al. 2015) and Feldman (1992) coronal abundances, were used for the analysis. By assuming a filling factor equal to unity, we calculated an emission measure (EM) and a lower limit of the electron density $\left(N_{\mathrm{e}}\right)$ in the region of the spire and in the region of the footpoint using $\mathrm{EM} \simeq 0.83\left\langle N_{\mathrm{e}}\right\rangle^{2} \Delta h$, where $\Delta h$ is the column depth of the plasma along the LOS. We integrated the DEM values over the temperature range $\log T[\mathrm{~K}]=5.8$ to $\log T[\mathrm{~K}]=6.7$ to calculate the $\mathrm{EM}$ 

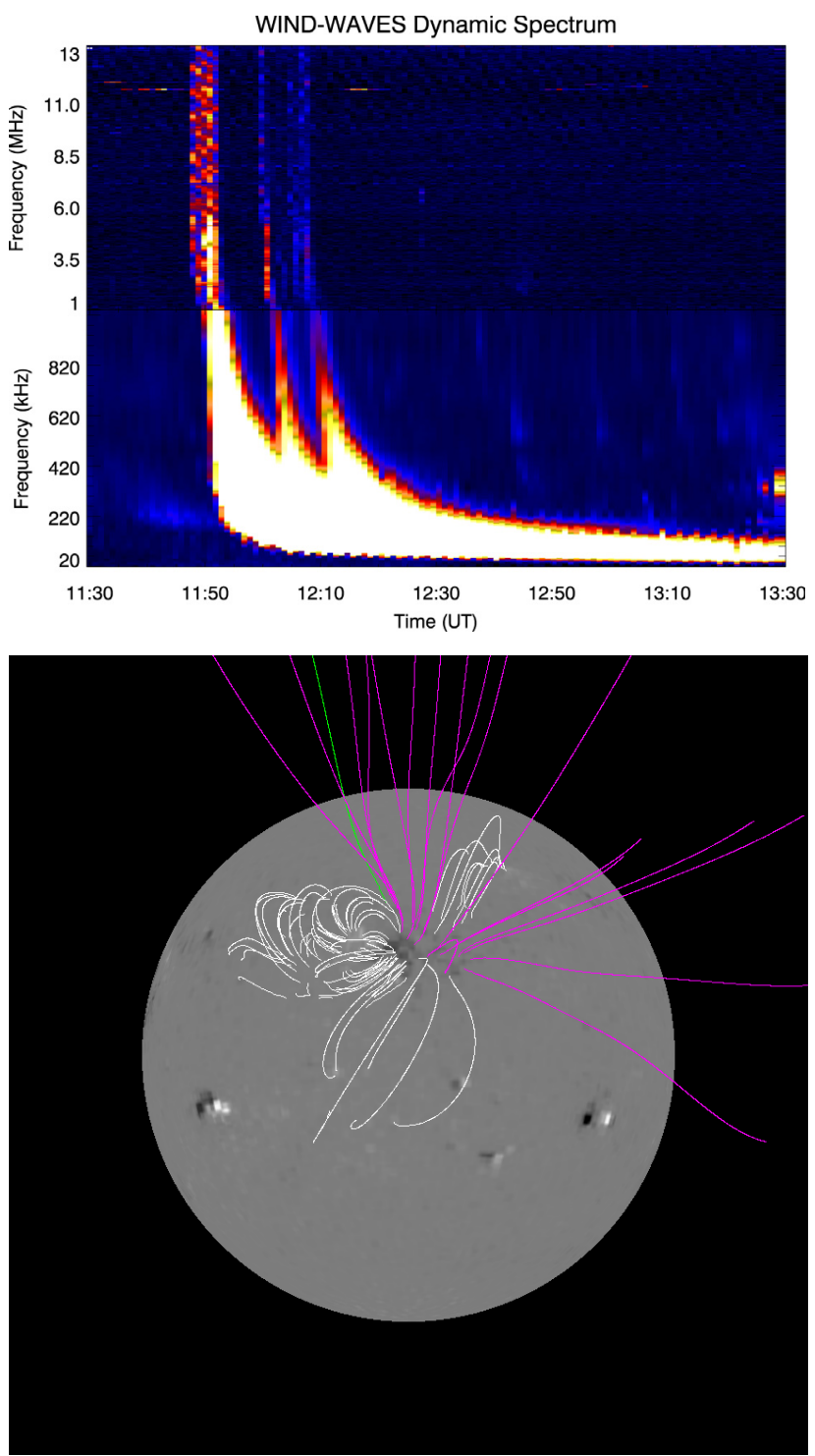

Fig. 11. Top panel: dynamic radio spectrum indicates the interplanetary nonthermal type-III radio burst, observed by WIND/WAVES at 11:50 UT. This burst is co-temporal with the jet evolution. The presence of the nonthermal type-III radio burst indicates the signature of particle acceleration. Bottom panel: PFSS extrapolation of active region at 12:04 UT. The white and pink lines indicate the closed and open magnetic structure in the active region and nearby region, respectively.

values. We obtained the column depth $\Delta h$ from the width of the spire (assuming cylindrical geometry) and the footpoint visible in the AIA $171 \AA$ images.

\subsection{August 02}

Figure 12 (top panels (a) and (b)) show the AIA $171 \AA$ images of the pre-jet phase at 17:03 UT and the jet phase at 17:25 UT respectively. We used two small regions, one at the footpoint of the jet and the other at the spire of the jet to calculate the LOS DEM. The regions are over-plotted as small black boxes in the Fig. 12 (top panel). We identify the same regions in the pre-jet phase at 17:03 UT and calculate the DEM curve. The plots (middle panels) show the DEM curves for the spire of the jet (red curve) and, at the same location, during the pre-jet phase (black curve). This DEM indicates that the temperature at
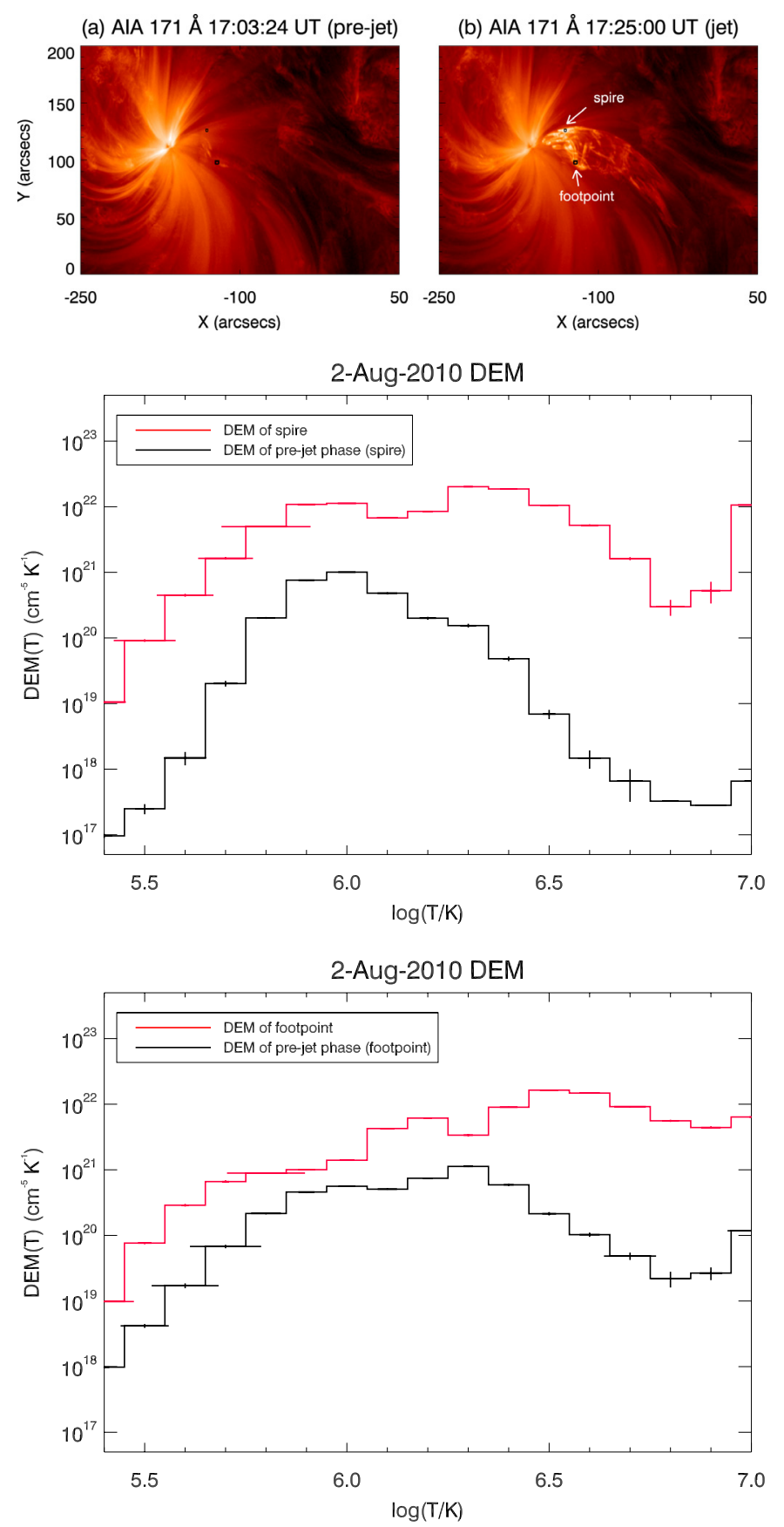

Fig. 12. Top panel: AIA $193 \AA$ image of a) pre-jet phase and b) jet. The two regions in the small boxes are used for the DEM analysis. Middle panel: shows the DEM curves for the spire of the jet (red curve) and, at the same region, during the pre-jet phase (black curve). Bottom panel: DEM curves for the footpoint of the jet (red curve) and in the same region, during the pre-jet phase (black curve).

the spire increased from $\log T[\mathrm{~K}]=6.0$ to $\log T[\mathrm{~K}]=6.3$ during the jet evolution. We note, however, the multi-thermal nature of the emission. The plots (bottom panel) show the DEM curves for the footpoint of the jet (red curve) and, in the same region, during the pre-jet phase (black curve). It is clear that, the temperature at the region of the footpoint is increased from $\log T[\mathrm{~K}]=6.3$ to $\log T[\mathrm{~K}]=6.5$. DEM measurements beyond $\log T[\mathrm{~K}]=7$ are not reliable due to the uncertainty in the contribution of emission lines to the AIA 94 and $131 \AA$ channels (Young \& Muglach 2014b). 


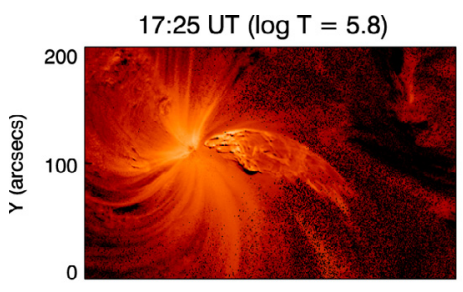

$(\log T=6.3)$
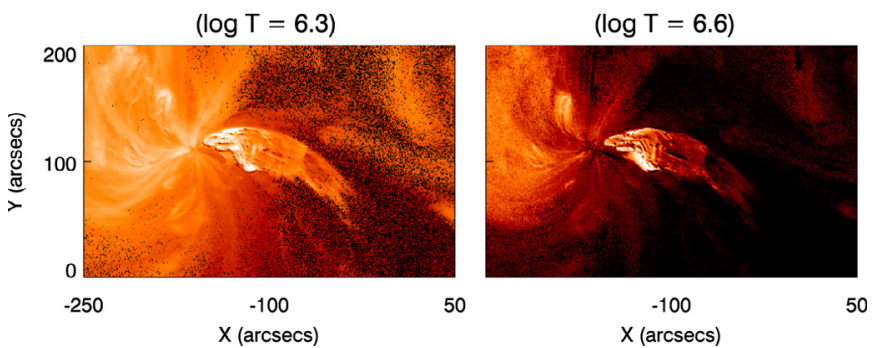

Fig. 13. DEM maps of the jet at different temperatures for the jet observed on 2010 August 02.

Figure 13 shows the DEM maps at different temperatures (at $\log T[\mathrm{~K}]=5.8,6.0,6.3$ and 6.6) at 17:25 UT. We investigated the temperature variation of coronal plasma at different locations. We observed that the spire and the footpoint during the jet evolution were at different temperatures and the results indicate that the spire has much more plasma at cooler temperatures than the footpoint. The temperature structure estimated for the small region from the DEM analysis (Fig. 12) shows consistent results with the DEM maps (Fig. 13) at different temperatures.

During the jet-phase, we found an emission measure of $\log \mathrm{EM}=28.6$ and a density of $N_{\mathrm{e}}=1.3 \times 10^{10} \mathrm{~cm}^{-3}$ at the location of the spire, and an emission measure of $\log \mathrm{EM}=28.6$ and a density of $N_{\mathrm{e}}=1.1 \times 10^{10} \mathrm{~cm}^{-3}$ at the location of the footpoint of the jet. During the pre-jet phase, we found an emission measure of $\log \mathrm{EM}=26.9$ and a density of $N_{\mathrm{e}}=1.8 \times 10^{9} \mathrm{~cm}^{-3}$ at the location of the spire, and an emission measure of $\log \mathrm{EM}=27.2$ and a density of $N_{\mathrm{e}}=2.4 \times 10^{9} \mathrm{~cm}^{-3}$ at the location of the footpoint of the jet. The results show that, the emission measure was increased by over one order of magnitude and the electron density was increased by a factor of ten during the jet evolution at the location of the spire and also at the location of the footpoint of the jet.

\subsection{March 02}

We performed a similar DEM analysis for the jet that had been observed on 2013 March 02. Figure 14 (top panels (a) and (b)) shows the AIA $171 \AA$ images of the pre-jet phase at 11:30 UT and jet phase at 11:53 UT, respectively. We used two small regions, one at the footpoint of jet and the other at the spire of the jet for LOS DEM calculation. The regions are over-plotted as small white boxes in Fig. 14 (top panel). The plots (middle panel) show the DEM curves for the spire of the jet (red curve) and, at the same region, during the pre-jet phase (black curve). This indicates that the temperature at the region of spire is increased from $T[\mathrm{~K}]=6.0$ to $T[\mathrm{~K}]=6.3$ during the jet evolution. The plots (bottom panel) show the DEM curves for the footpoint of the jet (red curve) and, at the same region, during the pre-jet phase (black curve). It is clear that the temperature at the footpoint increased from $\log T[\mathrm{~K}]=6.2$ to $\log T[\mathrm{~K}]=6.5$.

Figure 15 shows the DEM maps at different temperatures (at $\log T[\mathrm{~K}]=5.8,6.0,6.3$ and 6.6) at 11:53 UT. The temperature structure estimated for the small region from the DEM analysis
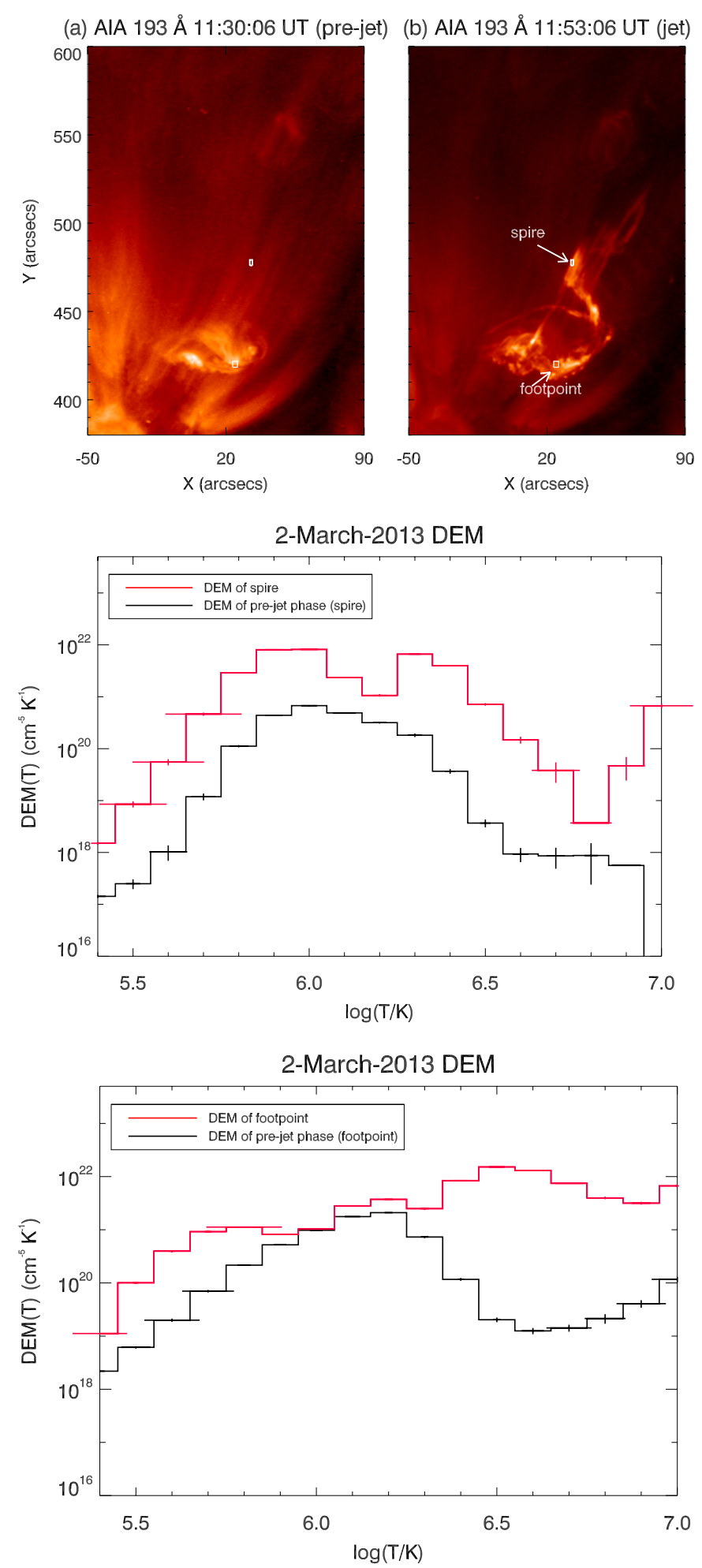

Fig. 14. Top panel: AIA $193 \AA$ image of a) pre-jet phase and b) jet. The two regions in the small boxes are used for the DEM analysis. Middle panel: DEM curves for the spire of the jet (red curve) and at the same region during the pre-jet phase (black curve). Bottom panel: DEM curves for the footpoint of the jet (red curve) and, at the same region, during the pre-jet phase (black curve).

(Fig. 14) shows consistent results with DEM maps (Fig. 15) at different temperatures.

During the jet-phase, we found an emission measure of $\log \mathrm{EM}=28.0$ and a density of $N_{\mathrm{e}}=8.6 \times 10^{9} \mathrm{~cm}^{-3}$ at the 

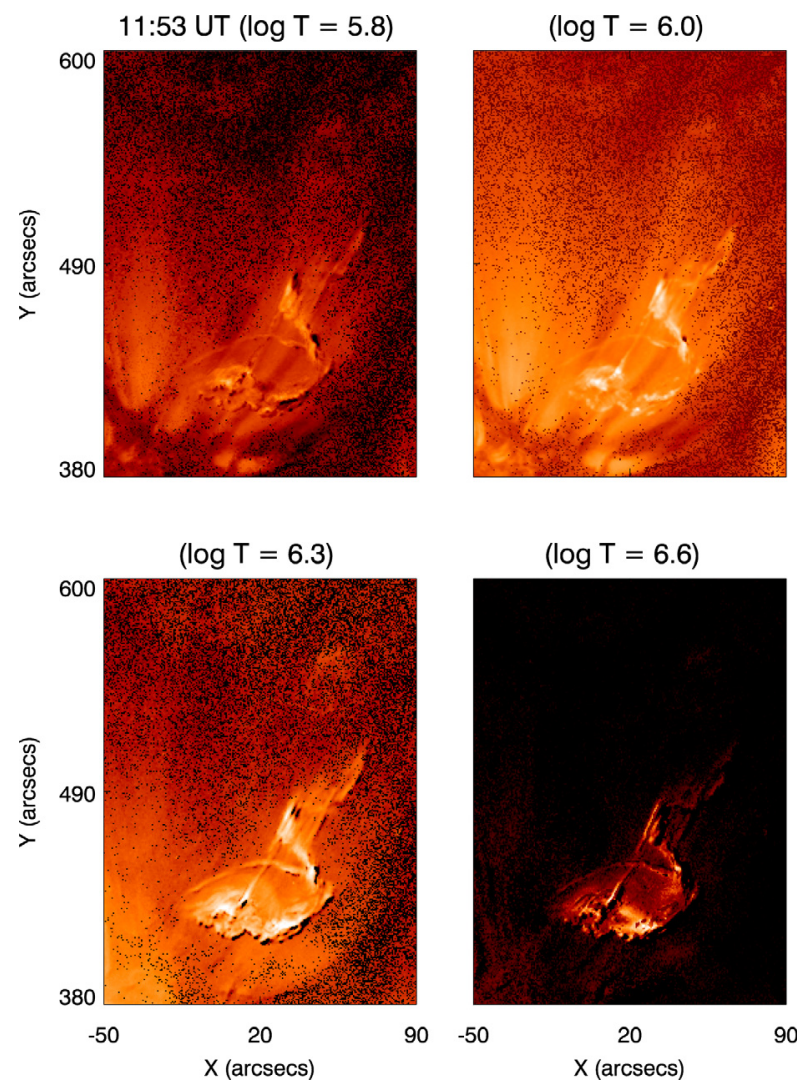

Fig. 15. DEM maps of the jet at different temperatures for the jet observed on 2013 March 02.

location of the spire, and an emission measure of $\log \mathrm{EM}=28.1$ and a density of $N_{\mathrm{e}}=8.4 \times 10^{9} \mathrm{~cm}^{-3}$ at the location of the footpoint of the jet. During the pre-jet phase, we found an emission measure of $\log \mathrm{EM}=26.8$ and a density of $N_{\mathrm{e}}=2.1 \times 10^{9} \mathrm{~cm}^{-3}$ at the location of the spire, and an emission measure of $\log \mathrm{EM}=27.3$ and a density of $N_{\mathrm{e}}=3.2 \times 10^{9} \mathrm{~cm}^{-3}$ at the location of the footpoint of the jet. The results show that, the emission measure increased by over one order of magnitude and the electron density increased by a factor of two during the jet evolution at the location of the spire. The emission measure was increased by a factor of ten and the electron density was increased by a factor of three at the location of the footpoint of the jet.

\section{Discussion and summary}

In this paper, we present a comprehensive study of multiwavelength observations of 20 active region jets observed during August 2010 - June 2013. We have used the observations recorded by SDO/AIA, SDO/HMI, RHESSI, WIND and GONG network. We have investigated the relationship of jets with $\mathrm{H} \alpha$ surges, nonthermal type-III radio burst, HXR emission and photospheric magnetic field configuration in the source region of all these jets. In addition, we have measured the general physical properties of the jets, such as lifetime and velocity. We have discussed two jet events in detail in Sects. 3 and 4, and the properties of other jets are discussed in the Appendix. Using the various filters of AIA, we have studied the temperature structure of these jets in their spires, as well as in their footpoints and have provided a lower limit on the electron densities. To the best of our knowledge, this is first comprehensive study of active region jets and their thermodynamic nature. Below we summarise the most important results.
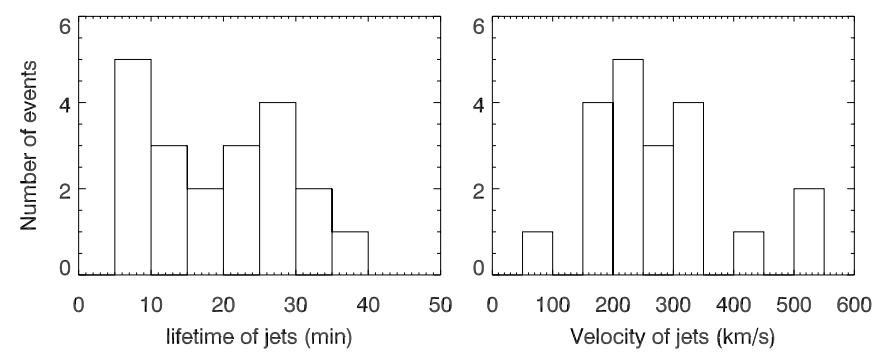

Fig. 16. Left panel: distribution of the lifetime of jets observed in the AIA 193 Å channel. Right panel: distribution of plane-of-sky velocities of jets, calculated from the time-distance plots.

Most of the jets originated from the western periphery of active regions close to sunspots. The lifetime (as noted from the AIA $193 \AA$ channel) ranged from 5 to 39 min with an average of $18 \mathrm{~min} .25 \%$ of the jets have their lifetime from 5 to $10 \mathrm{~min}$ and $20 \%$ of them have from 25 to $30 \mathrm{~min}$ (See Fig. 16). The velocities range from 87 to $532 \mathrm{~km} \mathrm{~s}^{-1}$ with an average of $271 \mathrm{~km} \mathrm{~s}^{-1}$. Of these, $25 \%$ of the jets have velocities from 200 to $250 \mathrm{~km} \mathrm{~s}^{-1}$ and other $20 \%$ have velocities from 300 to $350 \mathrm{~km} \mathrm{~s}^{-1}$ (See Fig. 16). These results are consistent with, and are within the range of values obtained by Shimojo et al. (1996) from their statistical study of 68 AR jets that were observed by SXT onboard Yohkoh. We investigated the cool-temperature component of the jets using $\mathrm{H} \alpha$ data from ground-based observatories. All of them were found to be associated with $\mathrm{H} \alpha$ surges at chromospheric temperatures.

The signature of an outward beam of mildly relativistic electrons from the jet source region to the outer corona and interplanetary space appears as a nonthermal type-III radio burst in the radio dynamic spectrum. To understand the relationship between nonthermal type-III radio bursts and jets, we compared the jet timings with burst timings using the dynamic radio spectrum that was obtained from WAVES/WIND. Of these, $85 \%$ of the nonthermal type-III radio bursts are found to be co-temporal with jets. We also confirm the position of these bursts by performing the PFSS analysis. This analysis showed the presence of open magnetic field lines in the source region, which indicates that jets were ejected in the direction of this open magnetic field structure. These observations show the signature of particle acceleration. The RHESSI observations showed the presence of HXR emission during the rising phase of jets. Here, $55 \%$ of the jets are found to be producing HXR emissions. Also, 10 out of 20 events showed that the jets originated in regions of flux cancellation and six in regions of flux emergence. Four events showed a flux emergence and then cancellation during the jet evolution.

We have performed a detailed analysis of the temperature distribution for the jets by using the computationally fast technique of regularized inversion developed by Hannah \& Kontar (2012). We found that, for most of the spires of the jets, the DEM peaked at around $\log T[\mathrm{~K}]=6.2 / 6.3(\sim 2 \mathrm{MK})$ (details in Table 1). The detailed analysis of two events showed that the temperature at the spire of the jets 1 and 2 increased from $\log T[\mathrm{~K}]=6.0$ to $\log T[\mathrm{~K}]=6.3$, and the temperature at the region of footpoint increased from $\log T[\mathrm{~K}]=6.3$ to $\log T[\mathrm{~K}]=6.5$ in the case of Jet 1 , and from $\log T[\mathrm{~K}]=6.2$ to $\log T[\mathrm{~K}]=6.5$ in the case of Jet 2 . The DEM values also showed consistency with DEM maps (Figs. 13 and 15) at different temperatures. These results show that the spires have plasma at cooler temperatures than at the footpoints. The observations of flux cancellation, the association 
with HXR emission, and emission of nonthermal type-III radio burst, suggest that the initiation and, therefore, heating is taking place at the base of the jets, and this is in agreement with the jet model proposed by Shimojo et al. (2001).

By assuming a filling factor equal to unity along with coronal abundances (Feldman 1992), we calculated the emission measure and a lower limit of the electron density. We observed that, there is an increase in the emission measure and electron density values during the jet evolution. In the case of Jet 1 , the emission measure was increased by over one order of magnitude and the electron density was increased by a factor of ten during the jet evolution at the location of the spire and also at the location of the footpoint of the jet. In the case of Jet 2, the emission measure was increased by over one order of magnitude and the electron density was increased by a factor of two during the jet evolution at the location of the spire. The emission measure was increased by a factor of ten and the electron density was increased by factor of three at the location of the footpoint of the jet.

These results are consistent with the results obtained by Culhane et al. (2007) and Kayshap et al. (2013) from their study of individual AR jets. Although they did not distinguish between the spire and footpoint region of the jet. The electron temperatures and DEMs derived in this study are also in agreement with the results obtained from the Fe XII line ratio diagnostics of a spectroscopic study of the recurrent AR jet (Mulay et al. in prep.).

The results described in this paper provide important and substantial constraints on the thermodynamics of jet plasma, which will be helpful to the theoretical modeling of jets.

Acknowledgements. We thank the referee for constructive and detailed comments which helped to improve this paper. Part of the work was done when one of the authors (SMM) was a Junior Research Fellow at Inter-University Centre of Astronomy and Astrophysics (IUCAA), India. S.M.M. and D.T. acknowledge support from DST under the Fast Track Scheme (SERB/F/3369/2012/2013). S.M.M. also ackowledges support from the Cambridge Trust, University of Cambridge, UK. H.E.M. and G.D.Z. acknowledge the support of STFC. AIA data are courtesy of SDO (NASA) and the AIA consortium. RHESSI work is supported by NASA contract NAS 5-98033. The authors thank the open data policy of WIND/WAVES instrument team. This work utilizes data obtained by the Global Oscillation Network Group (GONG) Program, managed by the National Solar Observatory, which is operated by AURA, Inc. under a cooperative agreement with the National Science Foundation. The data were acquired by instruments operated by the Big Bear Solar Observatory, High Altitude Observatory, Learmonth Solar Observatory, Udaipur Solar Observatory, Instituto de AstrofÃnsica de Canarias, and Cerro Tololo Interamerican Observatory.

\section{References}

Aschwanden, M. J., Boerner, P., Schrijver, C. J., \& Malanushenko, A. 2013, Sol. Phys., 283, 5

Bougeret, J.-L., Kaiser, M. L., Kellogg, P. J., et al. 1995, Space Sci. Rev., 71, 231

Canfield, R. C., Reardon, K. P., Leka, K. D., et al. 1996, ApJ, 464, 1016

Chae, J., Qiu, J., Wang, H., \& Goode, P. R. 1999, ApJ, 513, L75

Chandra, R., Gupta, G. R., Mulay, S., \& Tripathi, D. 2015, MNRAS, 446, 3741

Chandrashekhar, K., Morton, R. J., Banerjee, D., \& Gupta, G. R. 2014, A\&A, 562, A98

Chen, N., Ip, W.-H., \& Innes, D. 2013, ApJ, 769, 96

Cheng, X., Zhang, J., Saar, S. H., \& Ding, M. D. 2012, ApJ, 761, 62

Chifor, C., Young, P. R., Isobe, H., et al. 2008, A\&A, 481, L57
Culhane, L., Harra, L. K., Baker, D., et al. 2007, PASJ, 59, S751

Del Zanna, G. 2013, A\&A, 558, A73

Del Zanna, G., O’Dwyer, B., \& Mason, H. E. 2011, A\&A, 535, A46

Del Zanna, G., Dere, K. P., Young, P. R., Landi, E., \& Mason, H. E. 2015, A\&A, 582, A56

Doschek, G. A., Landi, E., Warren, H. P., \& Harra, L. K. 2010, ApJ, 710, 1806

Feldman, U. 1992, Phys. Scr., 46, 202

Glesener, L., Krucker, S., \& Lin, R. P. 2012, ApJ, 754, 9

Hannah, I. G., \& Kontar, E. P. 2012, A\&A, 539, A146

Hurford, G. J., Schmahl, E. J., Schwartz, R. A., et al. 2002, Sol. Phys., 210, 61

Hurlburt, N., Cheung, M., Schrijver, C., et al. 2012, Sol. Phys., 275, 67

Innes, D. E., Cameron, R. H., \& Solanki, S. K. 2011, A\&A, 531, L13

Jiang, Y. C., Chen, H. D., Li, K. J., Shen, Y. D., \& Yang, L. H. 2007, A\&A, 469, 331

Kayshap, P., Srivastava, A. K., \& Murawski, K. 2013, ApJ, 763, 24

Kundu, M. R., Raulin, J. P., Nitta, N., et al. 1995, ApJ, 447, L135

Lemen, J. R., Title, A. M., Akin, D. J., et al. 2012, Sol. Phys., 275, 17

Lin, R. P., Dennis, B. R., Hurford, G. J., et al. 2002, Sol. Phys., 210, 3

Madjarska, M. S. 2011, A\&A, 526, A19

Madjarska, M. S., Huang, Z., Doyle, J. G., \& Subramanian, S. 2012, A\&A, 545, A67

Matsui, Y., Yokoyama, T., Kitagawa, N., \& Imada, S. 2012, ApJ, 759, 15

Moreno-Insertis, F., Galsgaard, K., \& Ugarte-Urra, I. 2008, ApJ, 673, L211

Nisticò, G., Patsourakos, S., Bothmer, V., \& Zimbardo, G. 2011, Adv. Space Res., 48, 1490

Nitta, N. V., Mason, G. M., Wiedenbeck, M. E., et al. 2008, ApJ, 675, L125

O’Dwyer, B., Del Zanna, G., Mason, H. E., Weber, M. A., \& Tripathi, D. 2010, A\&A, 521, A21

Ogawara, Y., Takano, T., Kato, T., et al. 1991, Sol. Phys., 136, 1

Otruba, W. 1999, in Third Advances in Solar Physics Euroconference: Magnetic Fields and Oscillations, eds. B. Schmieder, A. Hofmann, \& J. Staude, ASP Conf. Ser. 184, 314

Parker, E. N. 1963, ApJS, 8, 177

Petschek, H. E. 1964, NASA SP, 50, 425

Pucci, S., Poletto, G., Sterling, A. C., \& Romoli, M. 2013, ApJ, 776, 16

Raulin, J. P., Kundu, M. R., Hudson, H. S., Nitta, N., \& Raoult, A. 1996, A\&A, 306, 299

Reid, H. A. S., \& Ratcliffe, H. 2014, RA\&A, 14, 773

Roy, J. R. 1973, Sol. Phys., 28, 95

Schatten, K. H., Wilcox, J. M., \& Ness, N. F. 1969, Sol. Phys., 6, 442

Scherrer, P. H., Schou, J., Bush, R. I., et al. 2012, Sol. Phys., 275, 207

Schmieder, B., Shibata, K., van Driel-Gesztelyi, L., \& Freeland, S. 1995, Sol. Phys., 156, 245

Schmieder, B., Guo, Y., Moreno-Insertis, F., et al. 2013, A\&A, 559, A1

Shibata, K. 2008, in COSPAR Meeting, 37th COSPAR Scientific Assembly, 37, 2858

Shibata, K., Ishido, Y., Acton, L. W., et al. 1992, PASJ, 44, L173

Shibata, K., Nitta, N., Matsumoto, R., et al. 1994, in X-ray solar physics from Yohkoh, eds. Y. Uchida, T. Watanabe, K. Shibata, \& H. S. Hudson (Tokyo: Univ. Academy Press), 29

Shibata, K., Nakamura, T., Matsumoto, T., et al. 2007, Science, 318, 1591

Shimojo, M., \& Shibata, K. 2000, ApJ, 542, 1100

Shimojo, M., Hashimoto, S., Shibata, K., et al. 1996, PASJ, 48, 123

Shimojo, M., Shibata, K., \& Harvey, K. L. 1998, Sol. Phys., 178, 379

Shimojo, M., Shibata, K., Yokoyama, T., \& Hori, K. 2001, ApJ, 550, 1051

Sun, J. Q., Cheng, X., Guo, Y., Ding, M. D., \& Li, Y. 2014, ApJ, 787, L27

Tsuneta, S., Acton, L., Bruner, M., et al. 1991, Sol. Phys., 136, 37

Ueno, S., Nagata, S., Kitai, R., \& Kurokawa, H. 2004, in The Solar-B Mission and the Forefront of Solar Physics, eds. T. Sakurai, \& T. Sekii, ASP Conf. Ser., 325, 319

Wang, Y.-M., Pick, M., \& Mason, G. M. 2006, ApJ, 639, 495

Yokoyama, T., \& Shibata, K. 1995, Nature, 375, 42

Yokoyama, T., \& Shibata, K. 1996, PASJ, 48, 353

Young, P. R., \& Muglach, K. 2014a, PASJ, 66, S12

Young, P. R., \& Muglach, K. 2014b, Sol. Phys., 289, 3313

Zhang, Q. M., \& Ji, H. S. 2014, A\&A, 567, A11

Zirin, H. 1970, Sky and Telescope, 39 

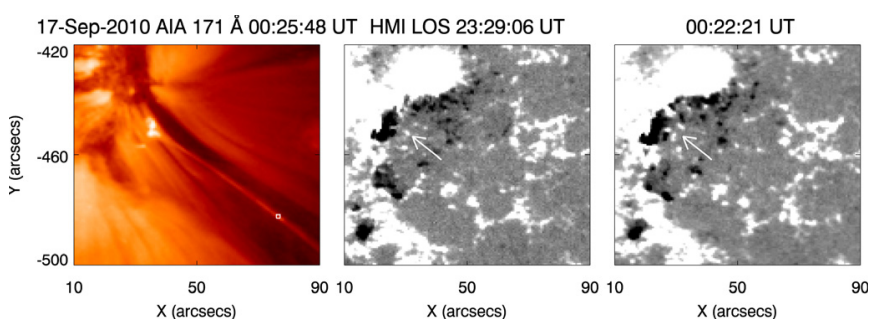

Fig. A.1. Jet 2: 2010 September 17 - Left panel: AIA $171 \AA$ A image of the jet observed on 2010 September 17. The jet appeared to evolve from the edge of the active region, 11106 (S20 W09), which was associated with a positive-polarity sunspot with anemone magnetic topology. A small-scale brightening was observed at the jet footpoint before the jet evolution and the jet evolved as a simple spire. The jet started its activity at 00:15 UT and lasted until 00:31 UT. Middle panel and right panel: the LOS HMI magnetogram image at 23:29 UT, before the jet evolution and at 00:22 UT, during the jet, respectively. The emergence of positive-polarity near the negative-polarity region is shown by white arrows. Later on, the small negative flux region cancelled with emerging positive-polarity. This magnetic activity lasted for a few hours and was co-temporal and co-spatial with the jet activity.
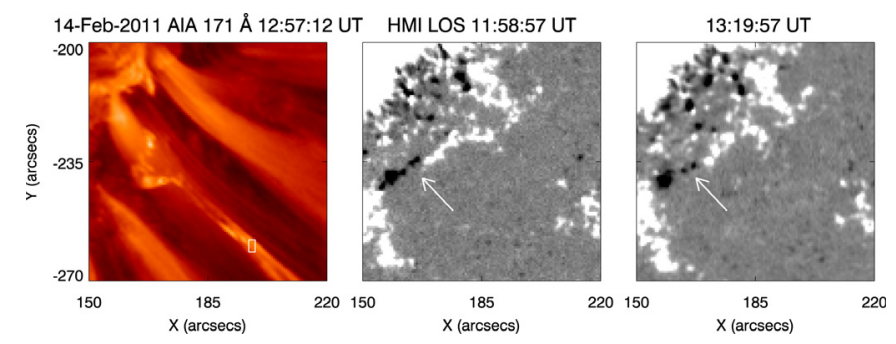

Fig. A.2. Jet 3: 2011 February 14 - Left panel: AIA $171 \AA$ A image of the jet, observed on 2011 February 14. The jet appeared to evolve from the edge of the active region, 11158 (S20 W17), which was associated with a positive-polarity sunspot. A small-scale brightening was observed at 12:58 UT at the jet footpoint before the jet evolution and the jet evolved as a simple spire. The jet started its activity at 12:58 UT and lasted until 13:02 UT. Middle panel and right panel: the LOS HMI magnetogram image at 11:58 UT, before the jet evolution, and at 13:19 UT, after the jet evolution, respectively. Negative flux cancellation was observed near the positive-polarity region shown by white arrows. This magnetic activity lasted for a few hours and was co-temporal and co-spatial with the jet activity.

\section{Appendix A: Information about all jets}

In this section, we discuss information about all AR jet events used in this multiwavelength analysis. Each of the figures for the jets show three panels that display the AIA $171 \AA$ image of a jet on the left, and in the middle and right panels, two HMI magnetograms during the jet evolution. A region in the small box over-plotted on AIA image is used for the DEM analysis.
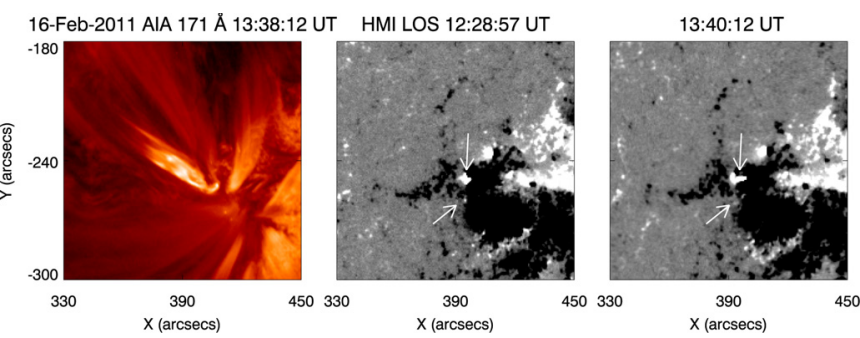

Fig. A.3. Jet 4: 2011 February 16 - Left panel: AIA $171 \AA$ A image of the jet observed on 2011 February 16. The recurrent jet appeared to evolve from the eastern edge of the active region, 11158 (S20 W41), which was associated with a negative-polarity sunspot. A small-scale brightening was observed at 13:32 UT at the jet footpoint before the jet evolution, and the jet evolved as a complex, multi-threaded spire. The observations show the continuous untwisting motion of the jet spire with radial motion. The jet started its activity at 13:24 UT, and lasted until 13:42 UT. Middle panel and right panel: the LOS HMI magnetogram image at 12:28 UT, before the jet evolution and at 13:40 UT, during the jet evolution, respectively. The positive flux emergence and cancellation was observed near the negative-polarity sunspot region shown by white arrows. This magnetic activity lasted for a few hours and was co-temporal and co-spatial with the jet activity.
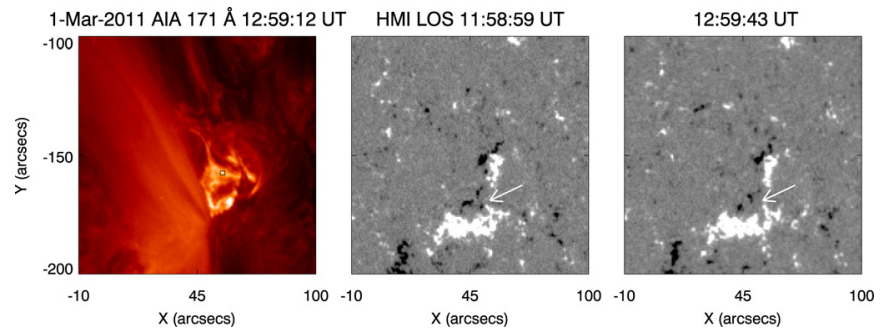

Fig. A.4. Jet 5: 2011 March 1 - Left panel: AIA $171 \AA$ image of the jet, observed on 2011 March 1. The jet appeared to evolve from the edge of the active region 11165 (S21 W00). A small-scale brightening was observed at 12:53 UT at the jet footpoint, before the jet evolution and the jet evolved as a complex, multi-threaded spire. The jet started its activity at 12:53 UT, and lasted until 13:18 UT. Middle panel and right panel: the LOS HMI magnetogram image at 11:58 UT before the jet evolution and at 12:59 UT, during the jet evolution, respectively. The negative flux cancellation with nearby positive-polarity region was observed and is shown by white arrows. This magnetic activity lasted for a few hours and was co-temporal and co-spatial with the jet activity. 

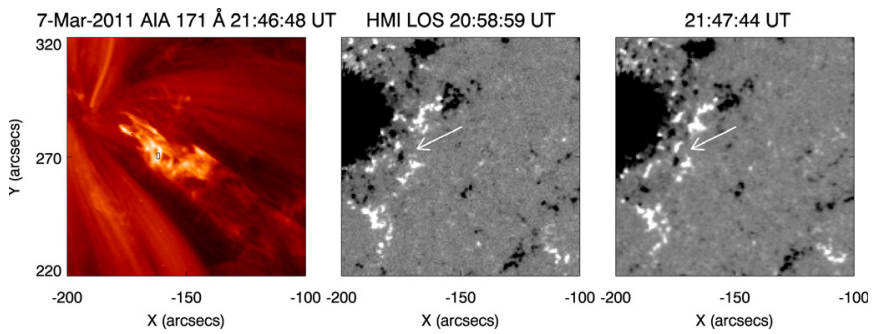

Fig. A.5. Jet 6: 2011 March 7 - Left panel: AIA $171 \AA$ image of the jet observed on 2011 March 7. The jet appeared to evolve from the edge of the active region, 11166 (N11 E13), which was associated with a negative-polarity sunspot with anemone magnetic topology. A smallscale brightening was observed at 21:33 UT at the jet footpoint before the jet evolution and the jet evolved as a complex, multi-threaded spire. The observations show the continuous untwisting motion of jet spire with radial motion. The jet started its activity at 21:33 UT and lasted until 22:12 UT. Middle panel and right panel: the LOS HMI magnetogram image at 20:58 UT, before the jet evolution, and at 21:47 UT, during the jet evolution, respectively. The positive flux emergence at the edge of the negative-polarity region was observed and is shown by white arrows.
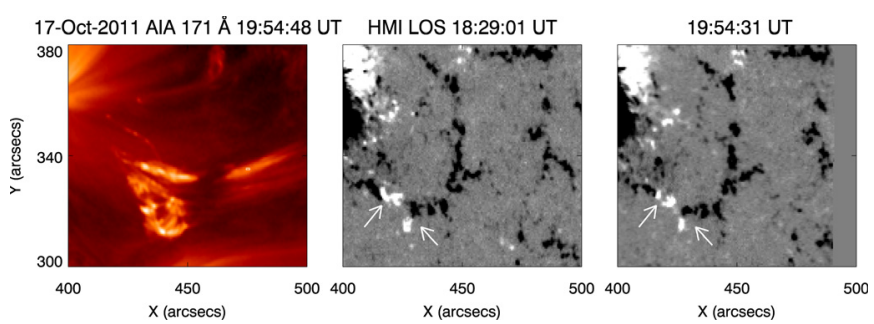

Fig. A.6. Jet 7: 2011 October 17 - Left panel: AIA $171 \AA$ Amage of the jet, observed on 2011 October 17. The jet appeared to evolve from the edge of the active region, 11314 (N28 W32), which was associated with a negative-polarity sunspot. A small-scale brightening was observed at 19:49 UT at the jet footpoint, before the jet evolution and the jet evolved as a complex, multi-threaded spire with inverted Y topology. The jet started its activity at 19:48 UT and lasted until 20:04 UT. Another similar jet was also observed at 20:07 UT in a nearby region. Middle panel and right panel: the LOS HMI magnetogram image at 18:29 UT, before the jet evolution, and at 18:54 UT, during the jet evolution, respectively. Flux cancellation was observed and is shown by white arrows. This magnetic activity lasted for a few hours and was co-temporal and co-spatial with the jet activity.

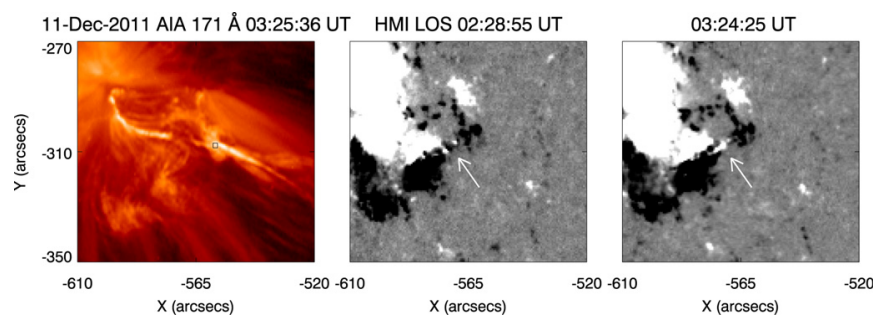

Fig. A.7. Jet 8: 2011 December 11 - Left panel: AIA 171 A image of the jet observed on 2011 December 11 . The jet appeared to evolve from the edge of the active region, 11374 (S17 E27), which was associated with a positive-polarity sunspot with anemone magnetic topology. A smallscale brightening was observed at a 03:21 UT at the jet footpoint, before the jet evolution. The jet evolved as a complex, multi-threaded spire. The jet started its activity at 03:21 UT and lasted until 03:26 UT. Middle panel and right panel: the LOS HMI magnetogram image at 02:28 UT, before the jet evolution, and at 03:24 UT, during the jet evolution, respectively. The negative-polarity cancellation followed by positive flux emergence at the edge of the negative-polarity region was observed and is shown by white arrows. This magnetic activity lasted for a few hours and was co-temporal and co-spatial with the jet activity.
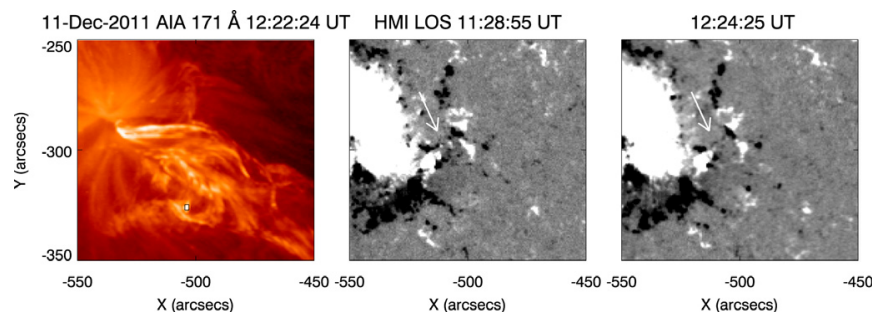

Fig. A.8. Jet 9: 2011 December 11 - Left panel: AIA $171 \AA$ image of the jet, observed on 2011 December 11. The jet appeared to evolve from the edge of the active region, 11374 (S17 E27), which was associated with a positive-polarity sunspot with anemone magnetic topology. A small-scale brightening was observed at 12:15 UT at the jet footpoint before the jet evolution and the jet evolved as a complex, multi-threaded spire. The observations show the continuous untwisting motion of jet spire. The jet started its activity at 12:15 UT, and lasted until 12:49 UT. Middle panel and right panel: the LOS HMI magnetogram image at 11:28 UT, before the jet evolution, and at 12:24 UT, during the jet evolution, respectively. The positive-polarity cancellation at the edge of the negative-polarity region was observed and is shown by white arrows. This magnetic activity lasted for a few hours and was co-temporal and co-spatial with the jet activity. 

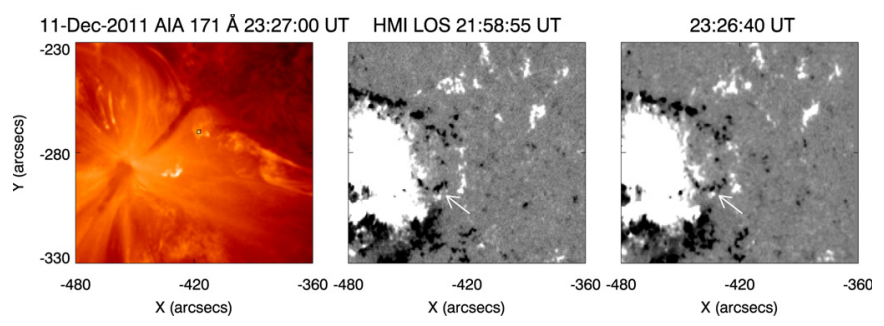

Fig. A.9. Jet 10: 2011 December 11 - Left panel: AIA $171 \AA$ image of the jet, observed on 2011 December 11. The jet appeared to evolve from the edge of the active region, 11374 (S17 E27), which was associated with a positive-polarity sunspot with anemone magnetic topology. A small-scale brightening was observed at 23:14 UT at the jet footpoint before the jet evolution and the jet evolved as a complex, multi-threaded spire. The observations show the continuous untwisting motion of jet spire. The jet started its activity at 23:14 UT and lasted until 23:34 UT. Middle panel and right panel: the LOS HMI magnetogram image at 21:58 UT, before the jet evolution, and at 12:24 UT, during the jet evolution, respectively. The positive-polarity cancellation at the edge of the negative-polarity region was observed and is shown by white arrows. This magnetic activity lasted for a few hours and was co-temporal and co-spatial with the jet activity.
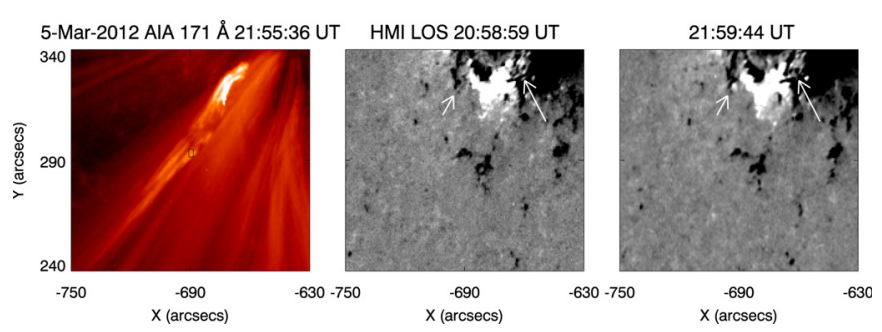

Fig. A.10. Jet 11: 2012 March 5 - Left panel: AIA $171 \AA ̊$ image of the jet, observed on 2012 March 5. The jet appeared to evolve from the edge of the active region, 11429 (N18 E41). A small-scale brightening was observed at 21:50 UT at the jet footpoint before the jet evolution and the jet evolved as a multi-threaded spire. A small loop is observed at the footpoint. The jet started its activity at 21:51 UT and lasted until 22:00 UT. Middle panel and right panel: the LOS HMI magnetogram image at 20:58 UT, before the jet evolution, and at 21:59 UT, during the jet evolution, respectively. The positive-polarity cancellation with nearby negative-polarity region was observed and is shown by white arrows. This magnetic activity lasted for a few hours and was co-temporal and co-spatial with the jet activity.
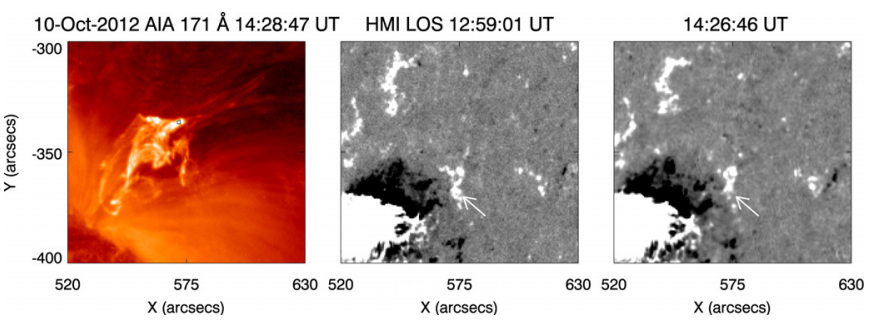

Fig. A.11. Jet 12: 2012 October 10 - Left panel: AIA 171 Å image of the jet, observed on 2012 October 10 . The jet appeared to evolve from the edge of the active region, 11585 (S20 W43). A small-scale brightening was observed at 14:26 UT at the jet footpoint before the jet evolution and the jet evolved as a multi-threaded spire. A small burst is observed at the footpoint. The jet started its activity at 14:21 UT and lasted until 14:45 UT. Middle panel and right panel: the LOS HMI magnetogram image at 12:59 UT, before the jet evolution, and at 14:26 UT, during the jet evolution, respectively. The positive-polarity cancellation with nearby negative-polarity region was observed and is shown by white arrows. This magnetic activity lasted for a few hours and was co-temporal and co-spatial with the jet activity.

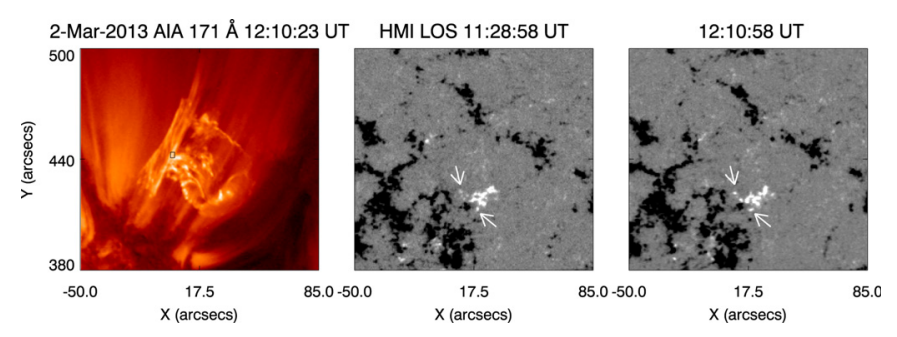

Fig. A.12. Jet 14: 2013 March 2 - Left panel: AIA $171 \AA$ image of the jet, observed on 2013 March 2. The jet appeared to evolve from the edge of the active region, 11681 (N17 W08). A small-scale brightening was observed at 12:03 UT at the jet footpoint before the jet evolution and the jet evolved as simple spire. The observations show the continuous untwisting motion of jet spire. The jet started its activity at 12:04 UT and lasted until 12:25 UT. Middle panel and right panel: the LOS HMI magnetogram image at 11:28 UT, before the jet evolution, and at 12:10 UT, during the jet evolution, respectively. The positivepolarity emergence at nearby negative-polarity region was observed and is shown by white arrows. This magnetic activity lasted for a few hours and was co-temporal and co-spatial with the jet activity. 

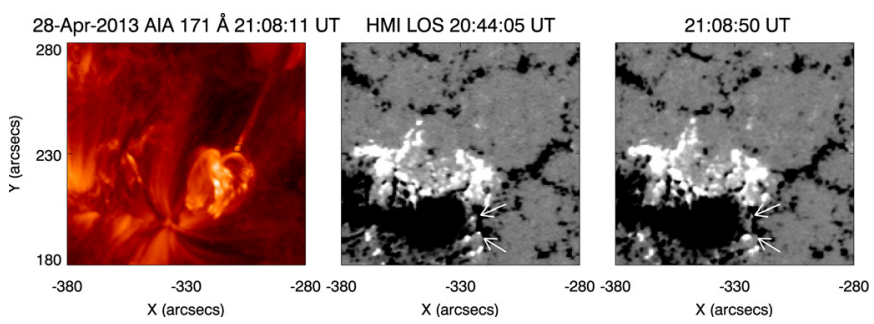

Fig. A.13. Jet 15: 2013 April 28 - Left panel: AIA $171 \AA$ A image of the jet, observed on 2013 April 28. The jet appeared to evolve from the edge of the active region, 11681 (N17 W08). A small-scale brightening was observed at 20:59 UT at the jet footpoint before the jet evolution and the jet evolved as a complex, multi-threaded spire. The observations show the continuous untwisting motion of jet spire. The jet started its activity at 20:59 UT and lasted until 21:11 UT. Middle panel and right panel: the LOS HMI magnetogram image at 20:44 UT, before the jet evolution, and at 21:08 UT, during the jet evolution respectively. The positivepolarity emergence and then cancellation observed near the negativepolarity region and is shown by white arrows. This magnetic activity lasted for a few hours and was co-temporal and co-spatial with the jet activity.

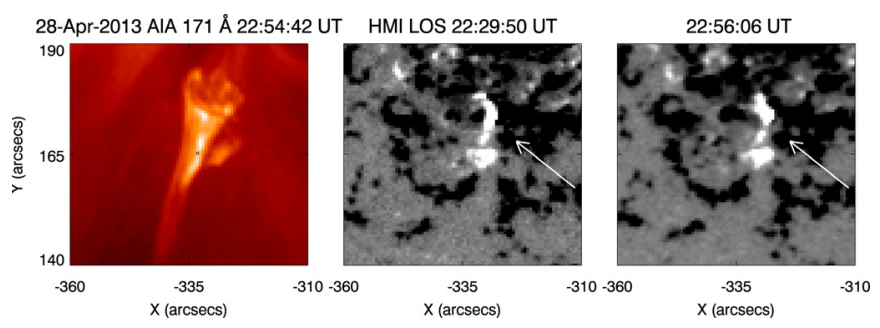

Fig. A.14. Jet 16: 2013 April 28 - Left panel: AIA $171 \AA$ A image of the jet, observed on 2013 April 28. The jet appeared to evolve from the edge of the active region, 11681 (N17 W08) which was associated with negative-polarity sunspot. A small-scale brightening was observed at 22:51 UT at the jet footpoint before the jet evolution and the jet evolved as multi-threaded spire with inverted $\mathrm{Y}$ topology of magnetic field. The jet started its activity at 22:51 UT and lasted until 22:59 UT. Middle panel and right panel: the LOS HMI magnetogram image at 22:29 UT, before the jet evolution, and at 22:56 UT, during the jet evolution, respectively. The positive-polarity emergence at nearby negative-polarity region was observed and is shown by white arrows. This magnetic activity lasted for a few hours and was co-temporal and co-spatial with the jet activity.
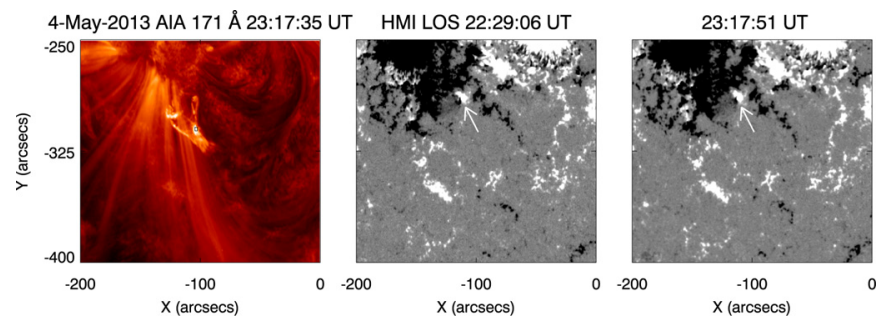

Fig. A.15. Jet 17: 2013 May 4 - Left panel: AIA $171 \AA$ image of the jet, observed on 2013 May 4. The jet appeared to evolve from the edge of the active region, 11734 (S19 W04). The jet was associated with a closed loop structure and a complex, multi-threaded jet was originated from one of the footpoints of loop. It has been observed that the jet plasma couldn't escape fully in the solar atmosphere, a part of it flows along the closed loop structure. The jet started its activity at 23:15 UT and lasted until 23:49 UT. Middle panel and right panel: the LOS HMI magnetogram image at 22:29 UT, before the jet evolution, and at 23:17 UT, during the jet evolution, respectively. The negativepolarity cancellation at nearby positive-polarity region was observed and is shown by white arrows. This magnetic activity lasted for a few hours and was co-temporal and co-spatial with the jet activity.
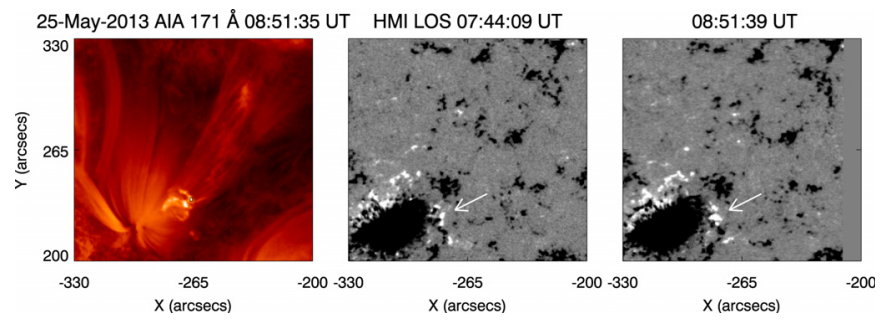

Fig. A.16. Jet 18: 2013 May 25 - Left panel: AIA $171 \AA$ image of the jet, observed on 2013 May 25. The jet appeared to evolve from the edge of the active region, 11748 (N12 W83). The jet was associated with a small loop at the footpoint and complex, multi-threaded jet was originated from it showing the untwisting nature. The jet started its activity at 08:45 UT and lasted until 08:53 UT. Middle panel and right panel: the LOS HMI magnetogram image at 07:44 UT, before the jet evolution, and at 08:51 UT, during the jet evolution, respectively. The positivepolarity emergence at the edge of the negative-polarity sunspot was observed and is shown by white arrows. This magnetic activity lasted for a few hours and was co-temporal and co-spatial with the jet activity. 


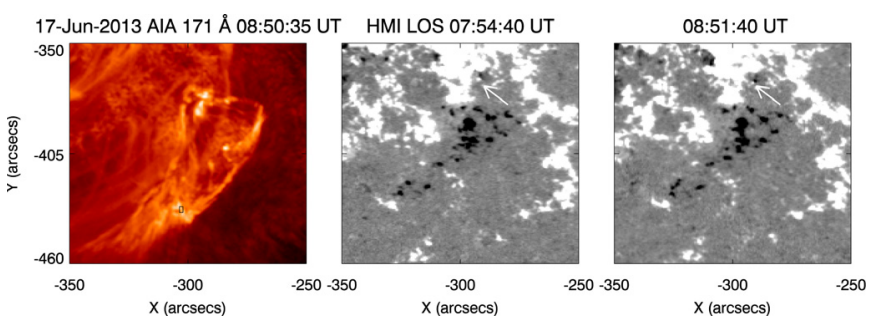

Fig. A.17. Jet 19: 2013 Jun 17 - Left panel: AIA $171 \AA$ image of the jet, observed on 2013 Jun 17. The jet appeared to evolve from the edge of the active region, 11770 (S13 E13). The jet was associated with a closed loop structure and a complex, multi-threaded jet was originated from one of the footpoints of loop showing its untwisting nature. It has been observed that the jet plasma couldn't escape fully in the solar atmosphere, a part of it flows along the closed loop structure. The jet started its activity at 08:40 UT and lasted until 09:06 UT. Middle panel and right panel: the LOS HMI magnetogram image at 07:54 UT, before the jet evolution, and at 08:51 UT, during the jet evolution, respectively. The positive-polarity cancellation at the nearby negative-polarity region was observed and is shown by white arrows. This magnetic activity lasted for a few hours and was co-temporal and co-spatial with the jet activity.
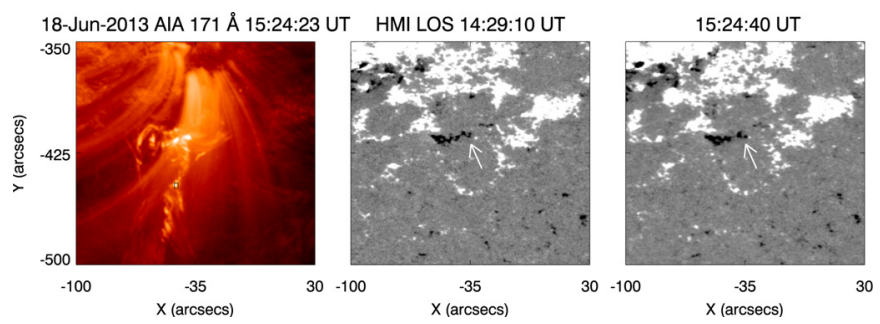

Fig. A.18. Jet 20: 2013 Jun 18 - Left panel: AIA $171 \AA$ image of the jet, observed on 2013 Jun 18 . The jet appeared to evolve from the edge of the active region, 11770 (S13 E13). The complex, multi-threaded jet was originated showing its untwisting nature. The jet started its activity at 15:13 UT and lasted until 15:39 UT. Middle panel and right panel: the LOS HMI magnetogram image at 14:29 UT, before the jet evolution, and at 15:24 UT, during the jet evolution, respectively. The positive-polarity cancellation at the nearby negative-polarity region was observed and is shown by white arrows. This magnetic activity lasted for a few hours and was co-temporal and co-spatial with the jet activity. 